\title{
Unsupervised Change Detection Using Fuzzy Topology-Based Majority Voting
}

\author{
Pan Shao ${ }^{1}\left(\mathbb{D}\right.$, Wenzhong $\mathrm{Shi}^{2}$, Zhewei Liu ${ }^{2} \mathbb{D}$ and Ting Dong ${ }^{1, *}$ \\ 1 College of Computer and Information Technology, China Three Gorges University, Yichang 443002, China; \\ panshao@whu.edu.cn \\ 2 Department of Land Surveying and Geo-Informatics, Smart Cities Research Institute, The Hong Kong \\ Polytechnic University, Hong Kong 999077, China; john.wz.shi@polyu.edu.hk (W.S.); \\ jackie.zw.liu@connect.polyu.hk (Z.L.) \\ * Correspondence: dongt@ctgu.edu.cn; Tel.: +86-071-7639-3153
}

Citation: Shao, P.; Shi, W.; Liu, Z.; Dong, T. Unsupervised Change Detection Using Fuzzy Topology-Based Majority Voting. Remote Sens. 2021, 13, 3171

https://doi.org/10.3390/rs13163171

Academic Editor: Carmine Serio

Received: 16 July 2021

Accepted: 8 August 2021

Published: 10 August 2021

Publisher's Note: MDPI stays neutral with regard to jurisdictional claims in published maps and institutional affiliations.

Copyright: (c) 2021 by the authors. Licensee MDPI, Basel, Switzerland. This article is an open access article distributed under the terms and conditions of the Creative Commons Attribution (CC BY) license (https:/ / creativecommons.org/licenses/by/ $4.0 /)$.

\begin{abstract}
Remote sensing change detection (CD) plays an important role in Earth observation. In this paper, we propose a novel fusion approach for unsupervised CD of multispectral remote sensing images, by introducing majority voting (MV) into fuzzy topological space (FTMV). The proposed FTMV approach consists of three principal stages: (1) the CD results of different difference images produced by the fuzzy $\mathrm{C}$-means algorithm are combined using a modified $\mathrm{MV}$, and an initial fusion CD map is obtained; (2) by using fuzzy topology theory, the initial fusion CD map is automatically partitioned into two parts: a weakly conflicting part and strongly conflicting part; (3) the weakly conflicting pixels that possess little or no conflict are assigned to the current class, while the pixel patterns with strong conflicts often misclassified are relabeled using the supported connectivity of fuzzy topology. FTMV can integrate the merits of different CD results and largely solve the conflicting problem during fusion. Experimental results on three real remote sensing images confirm the effectiveness and efficiency of the proposed method.
\end{abstract}

Keywords: remote sensing; unsupervised change detection; fuzzy topology; majority voting; conflict management

\section{Introduction}

Remote sensing change detection (CD) is a powerful process used to identify the changes on the Earth's surface using remote sensing images acquired of the same scene at different times. Such a process plays an important role in various fields, such as disaster assessment, urban studies, and environmental monitoring. A lot of CD methods have been designed and proposed in past decades [1]. These methods can be roughly divided into supervised and unsupervised groups, based on whether training samples are required [2]. Many pattern recognition techniques and methods, such as thresholding [2,3], fuzzy Cmeans algorithm (FCM) [4-6], support vector machine [7], wavelet transform [8], fuzzy topology [9], and information fusion [10], have been successfully applied to unsupervised $\mathrm{CD}$ for distinguishing the changed pixels from the unchanged ones. Information fusion, for example, is an effective way to enhance the performance of image analysis and has been widely used in remote sensing [10]. Usually, image fusion can be operated at three levels: the pixel level (also denoted as raw data level), feature level, and decision level [11].

Unsupervised CD methods are generally implemented via three steps [12]: (1) image preprocessing; (2) generating a difference image (DI) by comparing multitemporal remote sensing images, and (3) analyzing the DI to produce a CD map. Although most unsupervised CD methods produce only one DI for CD, there are some works that integrate multiple DIs. These works mainly perform DI fusion at two levels: pixel and decision. For the pixel-level fusion group, an ensemble of different DI images is integrated to generate a new better-quality DI, which is then used for CD, and the performance of CD can be 
therefore improved [13-17]. For the decision-level fusion group, the CD performance is enhanced by combining CD results from different DIs [8,11,18-23].

\subsection{Pixel-Level Fusion CD Methods}

Liu et al. [13] generated a better DI by combining the advantages of a log-ratio DI and a mean-ratio DI. The former is consistent with the real change but easily affected by noise, whereas the latter can suppress the noise, but is usually ambiguous. In order to make the change area more complete and preserve the edge information well, Zheng et al. [14] used a weighted average fusion strategy to integrate a mean-filtered subtraction DI and a median-filtered log-ratio DI. Bovolo [15] employed an image segmentation technique and multi-level change vector analysis to produce a fused DI, and thus the multiscale information of high-resolution images was utilized. To better characterize the change information, Inglada and Mercier [16] applied principal component analysis to combine multi-scale DI images, which were produced by Kullback-Leibler divergence based on pixel neighborhoods of increasing sizes. Zhuang et al. [17] produced a fused DI by constructing a hybrid feature vector (HFV) with magnitude and angle information to fully utilize the spectral vector.

\subsection{Decision-Level Fusion CD Methods}

Celik and Ma [8] exploited the inherent multiscale structure of the dual-tree complex wavelet transform to create multiple sub-band DI images, whose CD maps provided by thresholding were then integrated through intersection and union operations. Le HegaratMascle et al. [18] adopted the Dempster-Shafer theory (DS) to combine multi-index CD results, which can reduce both false alarm and misdetection levels. Du et al. [19] applied three classical decision-level fusion theories, namely DS, majority voting (MV), and fuzzy integral, to merge five types of typical DI images. Their experimental results show that these fusion techniques can enhance CD performance. Longbotham et al. [20] utilized both supervised and unsupervised algorithms to detect flooded areas, and then combined the best five detection results by means of MV to investigate the further improvement from decision fusion. Liu et al. [21] employed morphological profile to model the geometrical multiscale structure of changes, and used MV to combine the CD results obtained at different scales. Du et al. [11] proposed a two-stage sequential fusion framework for $\mathrm{CD}$ by integrating pan-sharpening and decision-level fusion, which makes full use of multi-resolution remotely sensed imagery and different fusion methods. Hedjam et al. developed [22] an iterative Bayesian-Markovian CD framework to combine the CD results from different thresholding algorithms, for obtaining more accurate $\mathrm{CD}$ result. By integrating $\mathrm{K}$-means clustering and adaptive $\mathrm{MV}$, Lv et al. [23] presented an adaptive fusion $\mathrm{CD}$ method (KMAMV), to enhance the performance of CD. In spite of the advantages of all the fusion $\mathrm{CD}$ methods mentioned above, a common drawback of these methods is recognized: they fail to fully consider the conflicts between different sources during fusion. Recently, in order to deal with the conflicting situations during fusion, a decision-level fusion CD method (termed as DSK) was proposed in [24] based on DS theory and indicator kriging [25]. In DSK the CD results from four different typical DIs are combined using DS first. The fusion CD result from DS is then partitioned into two parts: the weakly conflicting part and strongly conflicting part. Finally, the weakly conflicting pixels are labelled as the current class; and the strongly inconsistent pixels are reclassified using indicator kriging.

Although DSK can integrate the advantages of different CD results and significantly resolve the conflicting situations, it still has two main disadvantages: (1) DSK requires repetitive tuning of two crucial parameters to recognize the strongly conflicting pixels, which is quite laborious. (2) DSK needs to calculate experimental covariance functions for the changed and unchanged classes to use indicator kriging, which is time-consuming and leads to high computational complexity of DSK. 


\subsection{Our Method}

In order to maintain the advantages of DSK and overcome its disadvantages, this paper proposes a novel decision-level fusion $\mathrm{CD}$ approach for multiband remote sensing images, by introducing MV into fuzzy topological space (termed as FTMV). The proposed FTMV approach possesses the following attractive characteristics: (1) it is unsupervised; (2) it combines the CD results of different DIs; (3) it automatically recognizes the strongly conflicting pixels without any manual parameter tuning; (4) it proposes a simple but effective strategy to reclassify the strongly conflicting pixels based on support set connectivity (without computing experimental covariance functions); and (5) it can solve the conflicting situations during fusion to a large extent.

This paper is organized as follows. Section 2 describes the three datasets used for the experimental study. Section 3 details the proposed FTMV fusion CD method. Section 4 evaluates the effectiveness and efficiency of FTMV by analyzing experimental results. Finally, the discussion and conclusion of this paper are given in Sections 5 and 6.

\section{Study Site and Materials}

To evaluate the effectiveness and efficiency of the proposed FTMV fusion CD method, we considered three multispectral remote sensing datasets taken by different sensors with different changes and sizes, namely the Neimeng, Heilongjiang, and Hunan datasets (Figure 1).
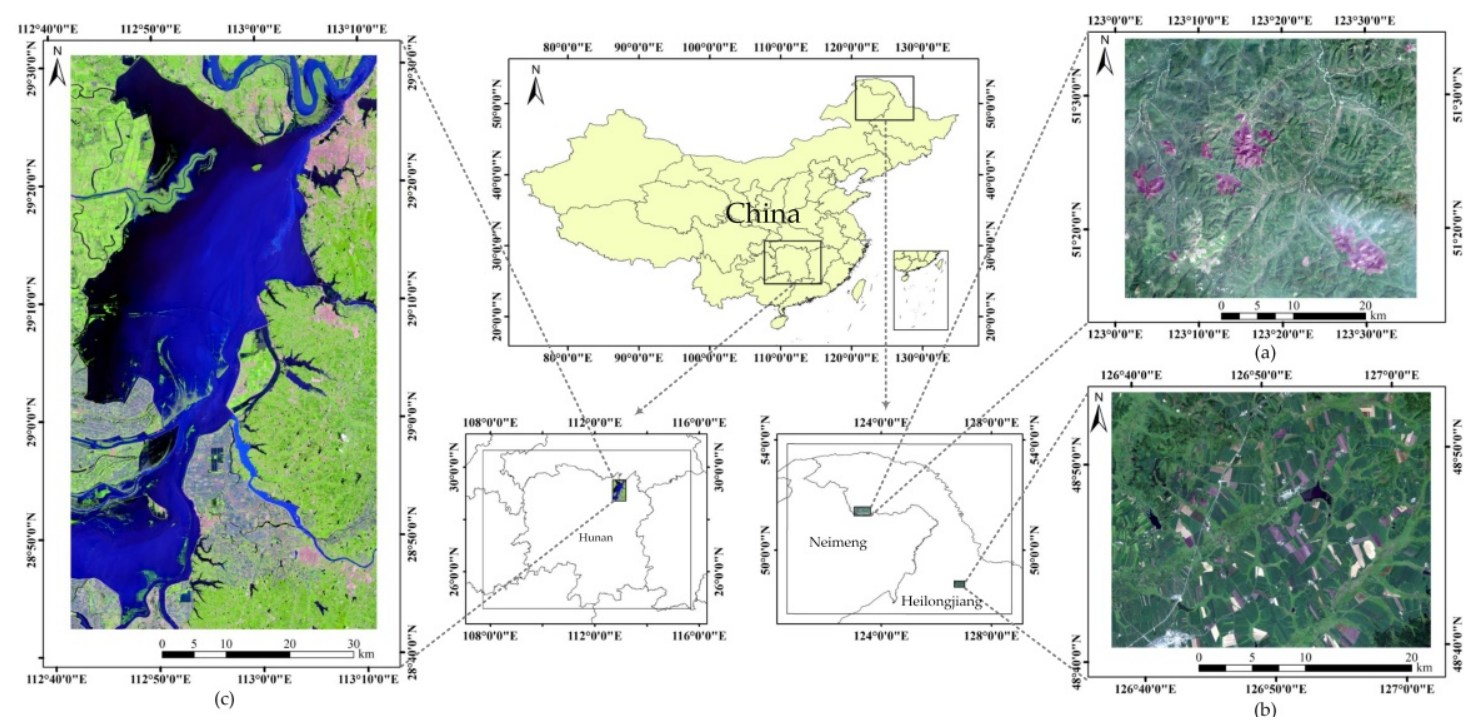

Figure 1. Location of the three used datasets in China: (a) Neimeng dataset, (b) Heilongjiang dataset, and (c) Hunan dataset.

The Neimeng dataset consists of two Landsat- 5 TM images acquired on the border of Neimeng and Heilongjiang Provinces on 22 August $2006\left(T_{1}\right)$ and 17 June $2011\left(T_{2}\right)$, respectively. A section of $1200 \times 1350$ pixels was selected for the experiments. These images display one main land cover types: forest. Between the two acquired times of the images, a wildfire (on 28 June 2010) destroyed a large portion of the forest in the studied region. Figure 2a-c exhibit the image taken in August, image taken in June, and their reference image of changes. The reference image (Figure 2c) of the Neimeng dataset was generated manually based on a detailed visual analysis of the two-temporal images (Figure 2a,b) using ENVI. For the other two datasets, the same method is used to create their reference images. 


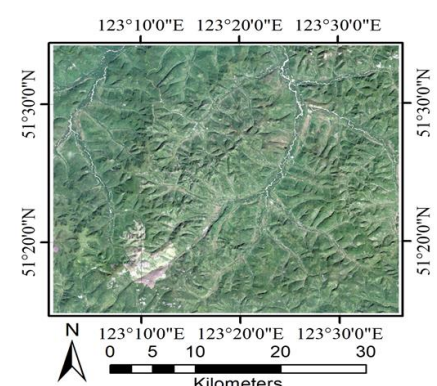

Legend

(a)

No Change

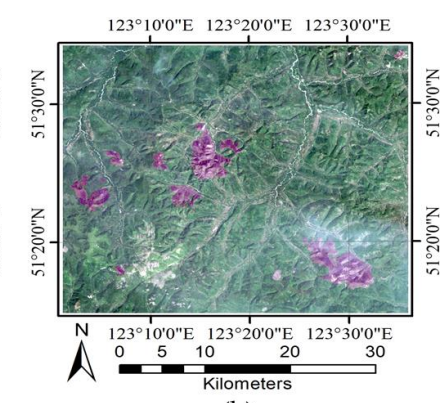

(b)

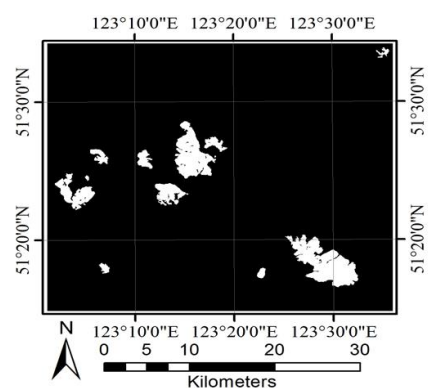

(c)

Figure 2. Neimeng dataset: (a) Image of 2006; (b) image of 2011; and (c) reference image.

The Heilongjiang dataset comprises two Landsat-7 ETM+ images taken in Heilongjiang Province on 11 August $2001\left(T_{1}\right)$ and 14 August $2002\left(T_{2}\right)$, respectively. A typical area with $800 \times 1000$ pixels is selected as the test site. There are roughly two land cover types in these images: farmland and woodland. The changes occurred in the considered area during the study period mainly due to the new crop planting. The images of 2001 and 2002 are displayed in Figure 3a,b, respectively, and their reference image is displayed in Figure 3c.

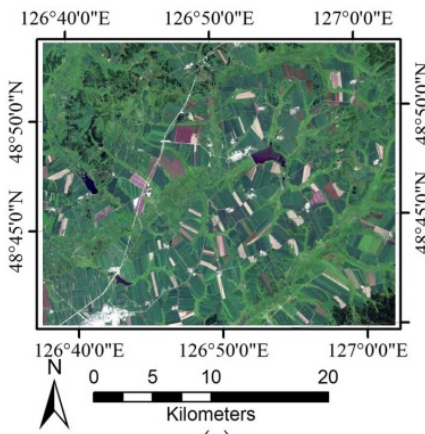
Legend (a)

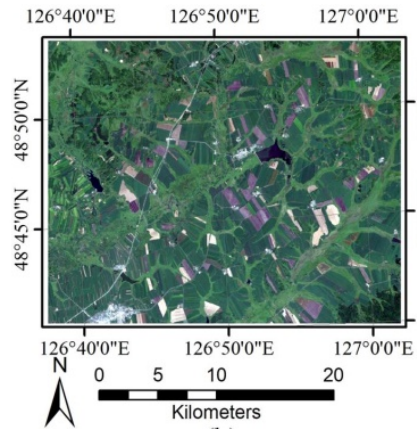

(b)

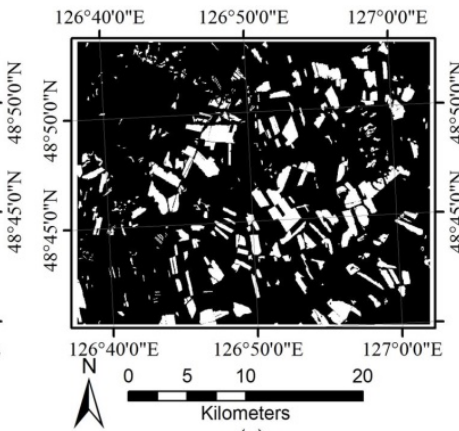

(c)

Figure 3. Heilongjiang dataset: (a) Image of 2001; (b) image of 2002; and (c) reference image.

The Hunan dataset is made up of two Landsat-8 Operational Land Imager (OLI) images acquired in Hunan Province on 17 September $2013\left(T_{1}\right)$ and 23 July $2016\left(T_{2}\right)$, respectively. These images cover an area with a size of $3000 \times 1600$ pixels, and include four main classes: building, farmland, water, and woodland. The new crop planting, persistent rain, and urban construction in the study area introduced multiple kinds of change. Figure 4a,b display the images of 2013 and 2016, respectively.

For the Hunan dataset, it was more challenging to perform CD and very difficult to produce its reference image for the entire area because of its diversity of change types and larger geographical size. The sampling technique is one of the most important methods to evaluate the accuracy of algorithms, which has been widely used in CD studies (e.g., [11,19,24,26-28]). As such, we used the sampling technique to evaluate the performance of $\mathrm{CD}$ on the Hunan dataset. To achieve this, fifteen image blocks (Figure 4c) were selected according to stratified sampling. In particular, in order to guarantee the rationality of sampling and the accuracy of evaluation results, the samples were obtained by the following steps: First, the study area was stratified based on change types to ensure that the samples contain all the types of change. Then, fifteen image blocks that had a balanced space distribution were selected based on the stratified result. The obtained image blocks thus possess a balanced distribution in space and contain all the types of change. Figure $4 \mathrm{c}$ displays the reference image for the Hunan dataset: white represents the changed areas, 
black represents the unchanged areas, and grey indicates that there is no reference image in the corresponding areas.

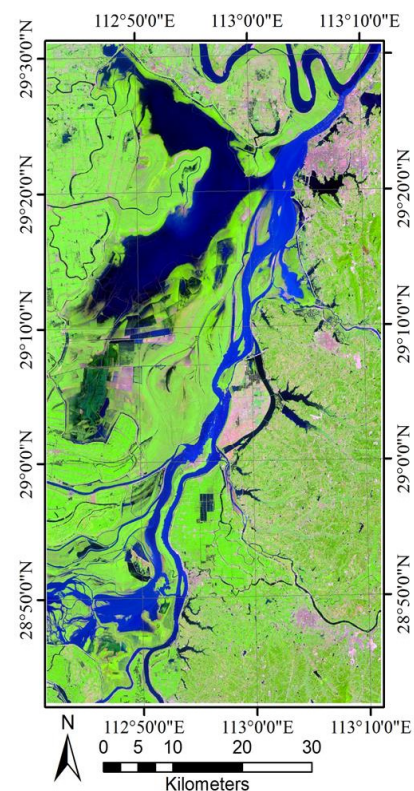

(a)

Legend

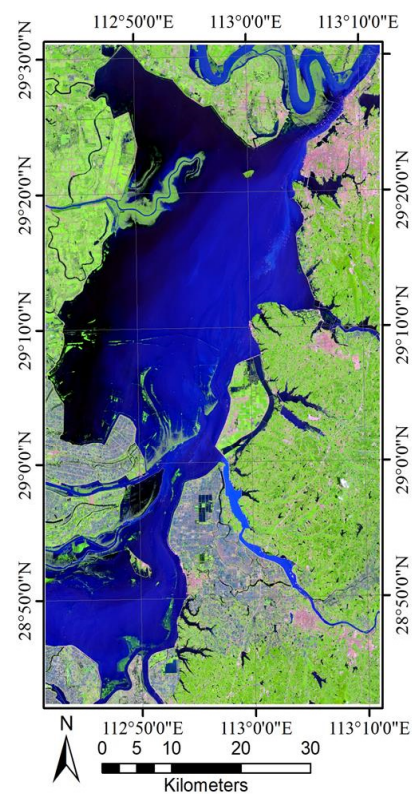

(b)

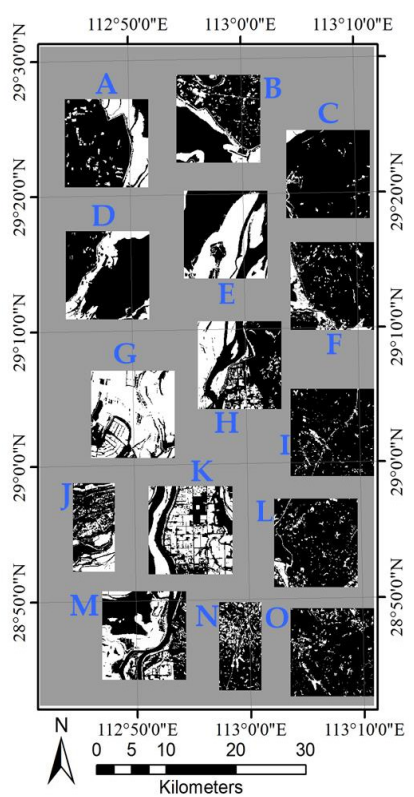

(c) No Change Change

No Reference Image for the Areas

Figure 4. Hunan dataset: (a) Image of 2013; (b) image of 2016; and (c) reference image.

The Neimeng and Heilongjiang datasets are used to test the effectiveness of the proposed FTMV CD method on single types of change (i.e., the forest change by a wildfire and farmland change by new crop planting). The Hunan dataset is used to test the effectiveness of FTMV for multiple types of change and larger geographical size, which is more challenging. To perform $\mathrm{CD}$, co-registration and (relative) radiometric correction are two necessary preprocessing operations which will render the two-temporal images comparable in both the spatial and spectral domains [12,29]. For each dataset, the $T_{2}$ image was first registered on the $T_{1}$ one, and then the relative radiometric correction was performed on $T_{2}$ image by referencing $T_{1}$ image using the histogram matching method.

\section{Proposed FTMV Fusion CD Method}

Let $X_{1}$ and $X_{2}$ denote two radiometrically corrected and co-registered multispectral remote sensing images acquired on the same ground area at two different times. A DI set with four elements providing complementary change information is produced for $X_{1}$ and $\mathrm{X}_{2}$, as done in [24]. The four DI images are generated by the change vector analysis (CVA), spectral correlation mapper (SCM), principal component analysis (PCA), and spectral gradient differencing (SGD), respectively. The four DI images are denoted as CVA, SCM, PCA, and SGD. More details of producing the four DIs can be found in [24]. It should be noted that the DI set used in FTMV can be adjusted in practical applications.

After obtaining the DI images, the proposed FTMV fusion algorithm is applied to analyze and combine them. As illustrated in Figure 5 and Table 1, the FTMV fusion CD method includes three principal stages: (1) initial fusion; (2) automatic partition; and (3) reclassification. In the following, Section 3.1 introduces the mathematical basis for the proposed FTMV method. Sections 3.2-3.4 detail the three stages of FTMV. 


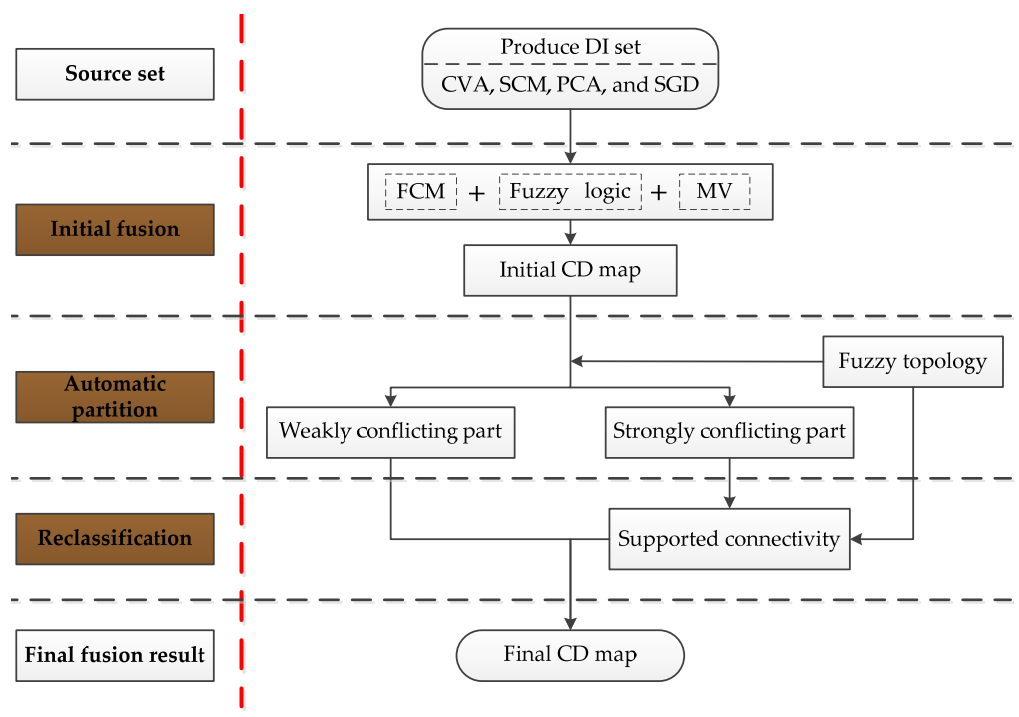

Figure 5. Flowchart of the proposed FTMV CD technique.

Table 1. The proposed FTMV CD technique.

\begin{tabular}{|c|c|c|c|}
\hline 1 & Initial fusion & $\begin{array}{l}1.1 \\
1.2 \\
1.3\end{array}$ & $\begin{array}{l}\text { Improve MV using fuzzy logic. } \\
\text { Analyze the DIs with FCM to obtain their CD results. } \\
\text { Generate an initial fusion CD map by combining the } \\
\text { DIs' CD results using the improved MV. }\end{array}$ \\
\hline 2 & Automatic partition & 2.1 & $\begin{array}{l}\text { Define the problem of partitioning the initial CD map } \\
\text { in fuzzy topological space. } \\
\text { Partition the initial CD map automatically using fuzzy } \\
\text { topology into two parts: strongly conflicting part and } \\
\text { weakly conflicting part. }\end{array}$ \\
\hline 3 & Reclassification & $\begin{array}{l}3.1 \\
3.2\end{array}$ & $\begin{array}{l}\text { Remain the weakly conflicting pixels. } \\
\text { Reclassify the strongly conflicting pixels through the } \\
\text { supported connectivity of fuzzy topology. }\end{array}$ \\
\hline
\end{tabular}

\subsection{Mathematical Basis for the Proposed FTMV}

This section introduces three key concepts: fuzzy set, fuzzy topology, and fuzzy topological space, which form the mathematical basis of the proposed FTMV. Fuzzy set is a generalization of the classical set by introducing the concept of partial membership. Let us consider a nonempty universal set $U$ and a complete lattice $I=[0,1]$. A fuzzy set $A$ on $U$ is a mapping $\mu_{A}(x): U \rightarrow I$, where $0 \leq \mu_{A}(x) \leq 1$ for all the elements $x$ in $U$. $\mu_{A}(x)$ is called the membership function of fuzzy set $A$.

Let $I^{U}$ be the family of all the fuzzy sets on $U$. That is, $I^{U}$ consists of all the mappings from $U$ to $I$. Let $\delta$ be a subset of $I^{U}$. Then, $\delta$ is called a fuzzy topology on $U$ and the pair $(U, \delta)$ is called a fuzzy topological space if $\delta$ satisfies the following three conditions [30]:

(1) $\varnothing, U \in \delta$.

(2) If $A, B \in \delta$, then $A \cap B \in \delta$.

(3) Let $\left\{A_{i}: i \in J\right\} \subseteq \delta$, where $J$ is an index set, then $\cup A_{i \in J} \in \delta$.

where $\varnothing$ and $U$ represent the empty set and whole set, and $\cup$ and $\cap$ denote the union operation and intersection operation of fuzzy sets.

In a traditional three-dimensional geometric space, a point is represented by a threedimensional vector $\left(x_{1}, x_{2}, x_{3}\right)$. That is, all the three dimensions $x_{1}, x_{2}$, and $x_{3}$ are used to describe the location information of the point. Different from this, for a triple $\left(x_{1}, x_{2}\right.$, $\left.\mu_{A}\left(x_{1}, x_{2}\right)\right)$ in a three-dimensional fuzzy topological space (Figure 6a), only the first two 
dimensions $x_{1}$ and $x_{2}$ describe the location of a point, whereas the third dimension represents the membership degree of the point belonging to the set $A$. As a consequent, the general fuzzy topological space contains two structures of space: membership (levelling) structure and location (neighbourhood) structure. The two structures correspond to the membership-value axis and the $x_{1}-0-x_{2}$ plane of the coordinate system used, respectively. Fuzzy topology thus is a suitable tool to study and analyze both the membership structure and neighbourhood structure of spaces.
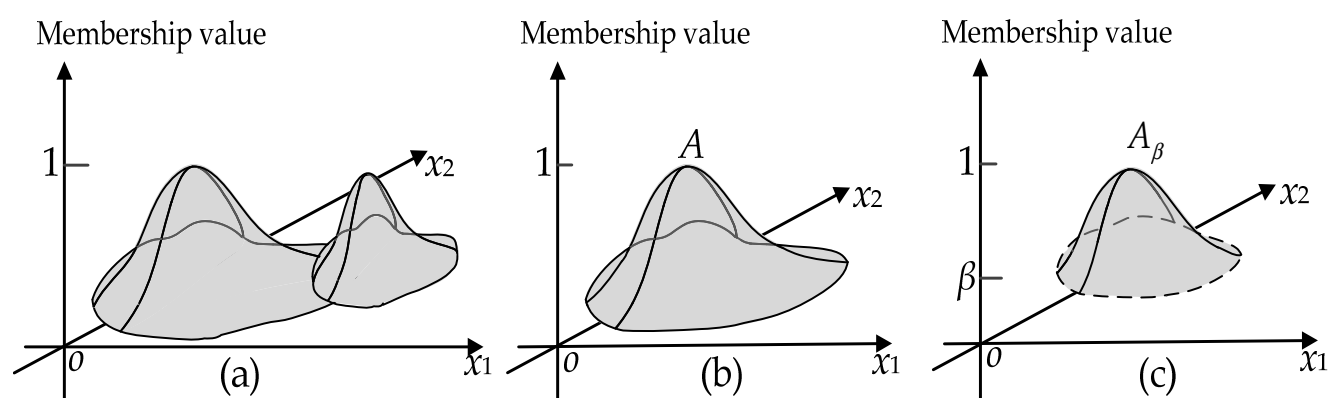

Figure 6. Examples of a fuzzy topological space and a level cut interior operator: (a) A simple example of a three-dimensional fuzzy topological space; (b) Fuzzy set $A$; and (c) Interior $A_{\beta}$ of fuzzy set $A$.

According to [30], fuzzy topology can be defined by the interior operator, which, in turn, can be defined by a suitable level cut. Let us consider a fuzzy set $A$ with a membership function $\mu_{A}(x)$; for a fixed value $\beta \in(0,1)$, we can define a level cut interior operator $A_{\beta}$ (Figure $6 \mathrm{~b}, \mathrm{c}$ ) as follows:

$$
A_{\beta}(x)=\left\{\begin{array}{ll}
A(x) & \mu_{A}(x)>\beta \\
0 & \mu_{A}(x) \leq \beta
\end{array} .\right.
$$

The level cut interior operator $A_{\beta}$ can induce a fuzzy topology (termed as LC fuzzy topology). Using LC fuzzy topology to analyze its membership function, a fuzzy set $A$ can be divided into two parts: interior $A^{\circ}$ and fuzzy boundary $\partial A$ :

$$
\left\{\begin{array}{l}
A^{o}=A_{\beta}=\left\{x \mid \mu_{A}(x)>\beta\right\} \\
\partial A=\left\{x \mid \beta \geq \mu_{A}(x)>0\right\}
\end{array} .\right.
$$

As an important characteristic of fuzzy topology, the connectivity of fuzzy spatial objects in a fuzzy topological space includes two types of structure: membership and location connectivity of points. Figure 7 presents two examples of fuzzy spatial objects in a two-dimensional fuzzy topological space: Object $A$ (Figure 7a) is membership connected but location disconnected, whereas Object $B$ (Figure $7 \mathrm{~b}$ ) is location connected but membership disconnected.
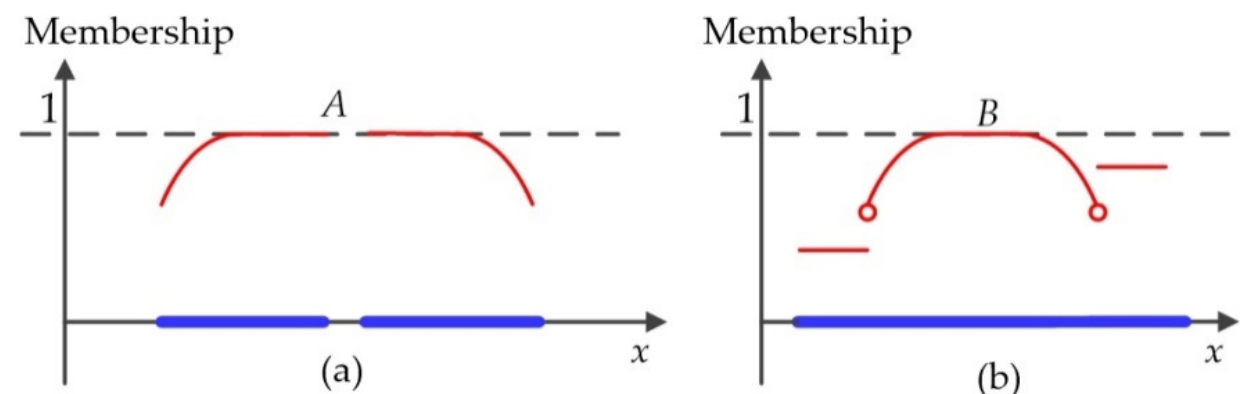

Figure 7. Two examples of fuzzy spatial objects in a two-dimensional fuzzy topological space: (a) An example of a membership connected but location disconnected object and (b) an example of a location connected but membership disconnected object. 
In this study, we define the decision-level fusion CD problem in terms of fuzzy topology, in which the changed and unchanged classes are regarded as fuzzy sets. In particular, the initial fusion and automatic partition stages of the proposed FTMV approach are realized by analyzing the membership structure of fuzzy topological space, whereas the reclassification stage is achieved by analyzing the location structure. We refer to [30] for more details on the fuzzy topological space.

\subsection{Initial Fusion}

MV is a simple but effective decision-level fusion technique and has been applied successfully in many fields. The main idea of MV is to combine the output results obtained from different data sources using the majority voting rule [11].

Let us consider an $n$-class $\left(C_{j}, j=1,2, \ldots, n\right)$ classification problem for which $m$ different data sources are available. For a given sample $x$, let $y_{i}(x)=\left\{y_{i 1}(x), y_{i 2}(x), \ldots, y_{i j}(x)\right.$, $\left.\ldots, y_{i n}(x)\right\}$ denote its classification result obtained based on source $S_{i}(i=1,2, \ldots, m, j=1$, $2, \ldots, n)$, where $y_{i j}(x)$ is a binary valued function, defined as follows:

$$
y_{i j}(x)= \begin{cases}1 & \text { if } x \text { is assigened to class } C_{j} \text { by } S_{i} \\ 0 & \text { otherwise }\end{cases}
$$

Let $V_{j}(x)$ denote the number of votes of sample $x$ to class $C_{j}(j=1,2, \ldots, n)$ received from the $m$ data sources. Then, $V_{j}(x)$ can be computed as follows:

$$
V_{j}(x)=\sum_{i=1}^{m} y_{i j}(x)
$$

According to the majority voting rule, the sample $x$ is assigned to the class $C_{k}$ with the largest number of votes as the consensus decision [31]:

$$
C_{k}=\arg _{j}\left\{\max \left\{V_{j}(x)\right\}\right\}, \quad j=1,2, \ldots, n .
$$

As shown in Equations (3)-(5), the conventional MV is based on the classical set theory, and makes decision by combining the binary valued class vectors (i.e., $\left.y_{i}(x)\right)$. However, using binary valued vectors for fusion may cause unreasonable fusion results in some cases.

\subsubsection{Improve MV Using Fuzzy Logic}

Let us consider a two-class $\left(C_{1}\right.$ and $\left.C_{2}\right)$ classification problem for which four sources $S_{i}(i=1,2,3,4)$ are available. Suppose that $d_{i j}(x)$ is the individual decision value (i.e., membership value or probability) according to source $S_{i}$ with respect to class $C_{j}(j=1$ or 2$)$ for a given sample $x$, and

$$
\begin{aligned}
& d_{11}(x)=d_{21}(x)=d_{31}(x)=0.49, d_{41}(x)=0.95 . \\
& d_{12}(x)=d_{22}(x)=d_{32}(x)=0.51, d_{42}(x)=0.05 .
\end{aligned}
$$

Then, the classification results $y_{i}(x)=\left\{y_{i 1}(x), y_{i 2}(x)\right\}$ can be obtained by comparing the decision values $d_{i 1}(x)$ and $d_{i 2}(x): y_{i 1}(x)=\left\{\begin{array}{ll}1 & \text { if } d_{i 1}(x) \geq d_{i 2}(x) \\ 0 & \text { otherwise }\end{array}\right.$ and $y_{i 2}=\left\{\begin{array}{ll}0 & y_{i 1}(x)=1 \\ 1 & y_{i 1}(x)=0\end{array}, i=1,2,3,4\right.$. Thus,

$$
\begin{aligned}
& y_{11}(x)=y_{21}(x)=y_{31}(x)=0, y_{41}(x)=1 . \\
& y_{12}(x)=y_{22}(x)=y_{32}(x)=1, y_{42}(x)=0 .
\end{aligned}
$$

According to Equations (4) and (5), for sample $x$, the final decision by MV will be in favour of class $C_{2}$ following the classification results $y_{1}(x), y_{2}(x)$, and $y_{3}(x)$ (from sources $S_{1}$, $S_{2}$, and $S_{3}$ ). Nevertheless, for sources $S_{1}, S_{2}$, and $S_{3}$, their decision values to classes $C_{1}$ and 
$C_{2}$ (0.49 and 0.51) are very close, causing their classification results to be very unreliable. On the contrary, the classification result from source $S_{4}$ is of high confidence (reflected by the large difference between the $d_{41}$ - and $d_{42}$-values). Therefore, it is more reasonable to assign $x$ to $C_{1}$ as the final decision by synthetically considering all four sources. The main reason for MV resulting in an unreasonable fusion result is that MV uses the binary (discrete) class label values $y_{i j}(x)$ for voting.

To resolve this problem, we can use the continuous decision values $d_{i j}(x)$ to replace the binary class label values $y_{i j}(x)$ for voting during MV fusion. This is because the difference between the continuous decision values can reflect the reliability in the source decision to some extent. By doing so, we will obtain continuous votes to classes $C_{1}$ and $C_{2}$, i.e., $V_{1}(x)=d_{11}(x)+d_{21}(x)+d_{31}(x)+d_{41}(x)=2.42$, and $V_{2}(x)=d_{12}(x)+d_{22}(x)+d_{32}(x)+d_{42}(x)=1.58$. According to Equation (5), MV will decide class $C_{1}$ and therefore yield a more reasonable fusion result.

Given the above analysis, we choose to use the continuous decision values for voting in $\mathrm{MV}$, to compensate its drawback in this study. As a consequence, Equation (4) is rewritten as follows:

$$
V_{j}(x)=\sum_{i=1}^{m} d_{i j}(x) j=1,2, \ldots, n,
$$

where $d_{i j}(x)$ represents the decision value of sample $x$ to class $C_{j}$ according to source $S_{i}$. In this work, the modified MV that performs fusion using continuous decision values is termed as fuzzy $M V$ (FMV).

\subsubsection{Analyze DI Images}

In our case, there are two classes to be identified: the change class $C_{c}$ and no-change class $C_{u}$. Four DI images serve as the four data sources: CVA, SCM, PCA, and SGD. Therefore, $m=4$ and $n=2$. We firstly analyze the DI images to determine each data source's individual decision values.

In general, the ranges of DI pixel values belonging to the changed and unchanged categories have overlap, and therefore the pixels from these two categories are not separable by sharp boundaries [5]. Analyzing DI bears some inherent uncertainty. Fuzzy clustering is an appropriate method to analyze the DI, because of its robust characteristics for uncertainty. The pixels of DI in fuzzy clustering are not assigned to class $C_{c}$ or class $C_{u}$, but to both classes with certain membership degrees [4].

In particular, we apply the widely used fuzzy clustering algorithm FCM to analyze the DIs for calculating their membership functions (also called fuzzy partition matrix). Then, the individual decision values $d_{i j}(x)$ for the four DI sources are derived according to the membership functions computed by FCM. More details of FCM can be found in [32].

\subsubsection{Generate an Initial Fusion CD Map by FMV}

Let $U_{i}=\left\{u_{i j}(x)\right\}$ denote the fuzzy membership functions obtained by FCM according to the $i$ th DI, $i=1,2,3,4, j=u, c$. Here $u_{i j}(x)$ represents the membership degree for pixel $x$ with respect to class $C_{j}$ obtained based on the $i$ th DI, and satisfies

$$
\left\{\begin{array}{l}
u_{i u}(x)+u_{i c}(x)=1 \\
0 \leq u_{i j}(x) \leq 1
\end{array} \quad j=u \text { or } c .\right.
$$

The individual decision values of each DI source are then defined using $U_{i}$. For a given pixel $x$, its decision value with respect to class $C_{j}$ from the $i$ th $\mathrm{DI}, d_{i j}(x)$, is given as follows:

$$
d_{i j}(x)=u_{i j}(x) \quad i=1,2,3,4, j=\text { u or } c .
$$

After obtaining $d_{i j}(x)(i=1,2,3,4, j=u$ or $c)$, the votes of pixel $x$ to class $C_{j}(j=u$ or $c)$, $V_{j}(x)$, are computed using Equation (6). An initial CD map is produced according to the 
majority voting rule expressed by Equation (5). Let $L(x)$ denote the class label of pixel $x$, and it is given by

$$
L(x)=\left\{\begin{array}{ll}
C_{u} & \text { if } V_{u}(x) \geq V_{c}(x) \\
C_{c} & \text { if } V_{u}(x)<V_{c}(x)
\end{array} .\right.
$$

\subsection{Automatic Partition}

The modified MV (i.e., FMV) can largely overcome the drawback of MV caused by using binary class label values, but it may still lead to unreliable CD results for the pixel patterns with strong conflicts. For example, let us consider a pixel $x_{A}$ with the following individual decision values:

$$
\begin{gathered}
d_{1 u}\left(x_{A}\right)=d_{2 u}\left(x_{A}\right)=0.03, d_{3 u}\left(x_{A}\right)=d_{4 u}\left(x_{A}\right)=0.98 . \\
d_{1 c}\left(x_{A}\right)=d_{2 c}\left(x_{A}\right)=0.97, d_{3 c}\left(x_{A}\right)=d_{4 c}\left(x_{A}\right)=0.02 .
\end{gathered}
$$

Then, using Equation (6), we can obtain $V_{u}\left(x_{A}\right)=2.02$ and $V_{c}\left(x_{A}\right)=1.98$, and the pixel $x_{A}$ is assigned to class $C_{u}$ according to Equation (9).

The decision values of pixel $x_{A}$ to classes $C_{u}$ and $C_{c}$ from every DI source (namely $d_{i u}\left(x_{A}\right)$ and $\left.d_{i c}\left(x_{A}\right)\right)$ are significantly different. Thus, the decision of each DI source at pixel $x_{A}$ has high confidence. However, the four sources at $x_{A}$ have strong conflicts: sources 1 and 2 are in favour of class $C_{c}$, whereas sources 3 and 4 in favour of class $C_{u}$. As a result, the votes of pixel $x_{A}$ to classes $C_{u}$ and $C_{c}\left(V_{u}\left(x_{A}\right)=2.02\right.$ and $\left.V_{c}\left(x_{A}\right)=1.98\right)$ are very close, which means the CD result of $x_{A}$ by FMV has high uncertainty.

The above analysis shows that, for the strongly conflicting pixels, FMV may lead to questionable CD results. Many pixels with strong conflicts are often misclassified in the initial fusion CD map created by FMV. To resolve this problem, this study adopts fuzzy topology to further improve the initial CD result. Firstly, the strongly conflicting pixels are determined by analyzing the membership structure of the FMV fusion result. Then, the determined pixel patterns are reclassified by reconstructing their neighbourhood structure, using the supported connectivity of fuzzy topology.

\subsubsection{Partition the Initial Fusion CD Map Conceptually}

Let $F S_{u}$ and $F S_{c}$ represent the sets that are composed of the unchanged and changed pixels in the initial CD map produced by FMV:

$$
\left\{\begin{array}{l}
F S_{u}=\left\{x \mid V_{u}(x) \geq V_{c}(x)\right\} \\
F S_{c}=\left\{x \mid V_{u}(x)<V_{c}(x)\right\}
\end{array} .\right.
$$

And let $\mathrm{Con}^{u}$ and $\mathrm{Con}^{c}$ denote the sets consisting of the strongly conflicting pixels in $F S_{u}$ and $F S_{c}$, respectively.

The difference between the votes $V_{u}(x)$ and $V_{c}(x)$ can reflect the four DI sources' conflict degree at pixel $x$ to a certain extent. The smaller the difference is, the more conflicting the four sources are. The conflict degree reaches the maximum when $V_{u}(x)$ and $V_{c}(x)$ are equal, i.e., $V_{u}(x)=V_{c}(x)=2$.

If we normalize $V_{j}(x)(j=u, c)$ using Equation (11), they will satisfy: $0 \leq V_{j}(x) \leq 1$ and $V_{u}(x)+V_{c}(x)=1$. Then, the normalized function $V_{j}(\cdot)(j=u$ or $c)$ can be regarded as a membership function of $F S_{j}$, and $F S_{j}$ can be regarded as a fuzzy set in fuzzy topological space.

$$
V_{j}(x)=\frac{V_{j}(x)}{V_{u}(x)+V_{c}(x)} j=u \text { or } c
$$

In addition, the difference between the normalized $V_{u}(x)$ and $V_{c}(x)$ can still reflect the conflict degree of the four DI sources at pixel $x$. Given that the smaller the difference, the more conflicting the sources, $0 \leq V_{j}(x) \leq 1$, and $V_{u}(x)+V_{c}(x)=1$, it is easy to obtain the following property. 
Property 1. For each pixel $x$, the closer the $V_{j}(x)$-value $(j=u$ or $c)$ is to 0.5 , the more conflicting the four DI sources are, and the conflict degree of sources will reach to the maximum when $V_{u}(x)=V_{c}(x)=0.5$.

Accordingly, the strongly conflicting pixel set $\operatorname{Con}{ }^{j}(j=u$ or $c)$ can be determined by analyzing the membership structure of the fuzzy set $F S_{j}$ using fuzzy topology. In particular, we use the LC fuzzy topology (Section 3.1) to analyze the fuzzy set $F S_{j}$, which is partitioned conceptually into two parts: interior $F S_{j}{ }^{0}$ and fuzzy boundary $\partial F S_{j}$ :

$$
\left\{\begin{array}{l}
F S_{j}^{o}=\left(F S_{j}\right)_{\beta_{j}}=\left\{x \mid V_{j}(x)>\beta_{j}\right\} \\
\partial F S_{j}=\left\{x \mid \beta_{j} \geq V_{j}(x)>0.5\right\}
\end{array},\right.
$$

where $\beta_{j}$ is a constant and $\beta_{j} \in(0.5,1)$. Obviously, the $V_{j}(x)$-values of the pixels in the fuzzy boundary $\partial F S_{j}$ are close to 0.5 . According to Property 1 , the pixels in $\partial F S_{j}$ are of strong conflicts. Thus, we use the fuzzy boundary $\partial F S_{j}$ to define the strongly conflicting pixel set Con' $^{\text {: }}$

$$
\operatorname{Con}^{j}=\partial F S_{j} \quad j=u \text { or } c .
$$

As a result, the initial CD map yielded by FMV is conceptually divided into two parts: strongly conflicting part $\mathrm{Con}^{u} \cup \mathrm{Con}^{c}$ and weakly conflicting part $F S_{u}{ }^{o} \cup F S_{c}{ }^{o}$.

\subsubsection{Determine the Optimal Threshold $\beta_{j}$}

The key to recognizing the strongly conflicting pixels is to determine the optimum threshold $\beta_{j}$ for $F S_{j}, j=u$ or $c$. An automatic and adaptive method is proposed in this study for searching the optimum $\beta_{j}$. As $0.5<\beta_{j}<1$, the search range is set as $(0.5,1)$. Two main factors need to be taken into consideration to set the search pace: (1) The optimum threshold may be missed if the search pace is too large; (2) the computational complexity may be too high if the search pace is very small. Considering both the two factors, we propose setting the searching pace as 0.05 , and search the optimum $\beta_{j}$ in the candidate set $C=\{0.55,0.60,0.65,0.70,0.75,0.80,0.85,0.90\}$.

According to Property 1 , the closer the $V_{j}(x)$-value is to 1 , the weaker the conflict and the more reliable the FMV CD result of pixel $x$. From this perspective, the $\beta_{j}$-value should be as large as possible. On the other hand, the larger the $\beta_{j}$-value is, the more pixels the strongly conflicting pixel set $\mathrm{Con}^{j}$ has. However, the final $\mathrm{CD}$ accuracy will be affected negatively if there are too many recognized strongly conflicting pixels in $\mathrm{Con}^{j}$. In general, the uncertain pixels likely to be misclassified in DI account for about $15 \%$ of the total pixels [7]. Denote the upper bound of the percentage of the strongly conflicting pixels in $F S_{c}$ by $R a_{c}$, and that in $F S_{u}$ by $R a_{u}$. A higher $R a_{c}$ or $R a_{u}$ value helps to recognize the strongly conflicting pixels in DI. However, a higher $R a_{c}$ and $R a_{u}$ also may increase missed detection errors and false alarm errors, respectively, in the subsequent reclassification. The meanings of missed detection errors and false alarm errors can be seen in Section 4.1. In most cases, the no-change area in DI is far larger than the change area. Thus, a higher $R a_{c}$ value for $F S_{c}$ is more risky and may lead to serious missed detection errors, while a higher $R a_{u}$ value for $F S_{u}$ is more beneficial for recognizing the strongly conflicting pixels. Accordingly, $R a_{c}$ is set to $10 \%$ and $R a_{u}$ is set to $20 \%$.

Given the above analysis, we present an automatic and adaptive method to search the optimum $\beta_{j}$ (Figure 8), termed as AAM, which consists of three main steps:

Step 1: Calculate the ratio set $R=\left\{R_{l} \mid R_{l}=n_{l} / n\right\}$ and set $l=1$. $n$ and $n_{l}$ stand for the numbers of the pixels in $F S_{j}$ and the set $\left\{x \mid 0.5<V_{j}(x)<c_{l}\right\}$, respectively. $c_{l}=0.5+0.05 \times l$ belongs to the candidate set $C, l=1,2,3,4,5,6,7,8$.

Step 2: If $R_{l} \geq R a_{j}\left(j=u\right.$ or $c$ ), then stop and let $\beta_{j}=c_{l-1}$, otherwise go to Step 3. Here $R a_{u}=20 \%, R a_{c}=10 \%$, and $c_{0}=0.5$.

Step 3: If $c_{l}=0.90$ then stop and let $\beta_{j}=0.90$, otherwise set $l=l+1$ and go to Step 2 . 


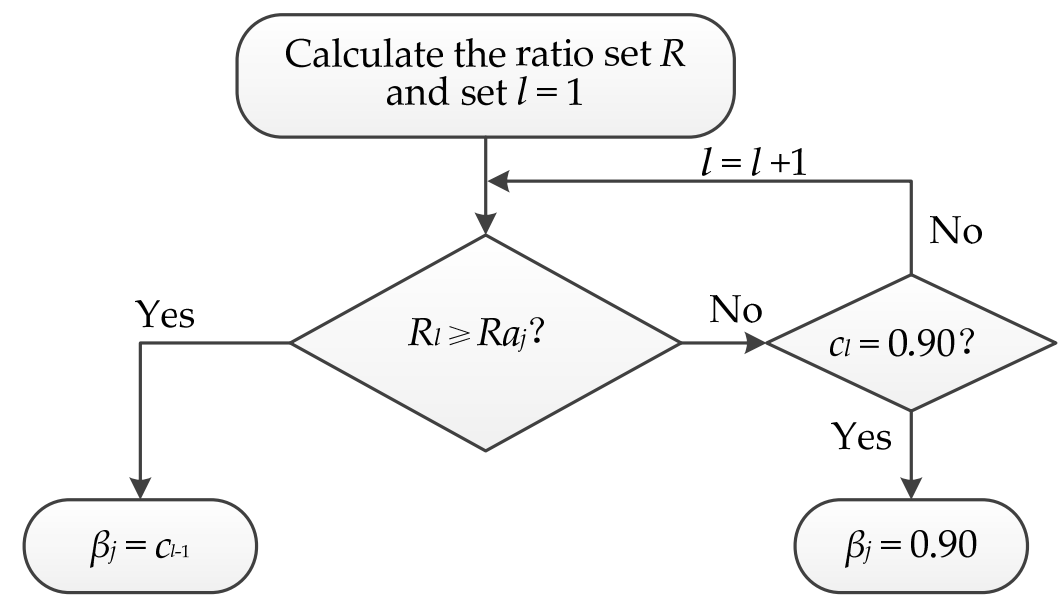

Figure 8. Flowchart of searching the optimal $\beta_{j}$.

The proposed searching approach AAM is to find the maximum $c_{l}$ that satisfies $R_{l}<R a_{j}$ from the candidate set $C$. The found $c_{l}$ can ensure that 1$)$ the $\beta_{j}$-value is as large as possible and 2) the recognized strongly conflicting pixels are within a reasonable range.

\subsection{Reclassification}

The initial fusion CD map by FMV has been partitioned into two parts in Section 3.3 by analyzing the membership structure of the fuzzy set $F S_{j}(j=u$ and $c)$ : strongly conflicting part $\operatorname{Con}^{u} \cup \operatorname{Con}^{c}$ and weakly conflicting part $F S_{u}^{o} \cup F S_{c}^{o}$. The pixels in the weakly conflicting part have little or no conflict. FMV has a good performance on classifying such pixels, so their CD results by FMV are taken as their final detection results. That is, the pixels in $F S_{\mathcal{C}}^{o}$ and $F S_{u}^{o}$ are finally assigned to classes $C_{c}$ and $C_{u}$, respectively. For the pixels in $\operatorname{Con}^{u} \cup \operatorname{Con}^{c}$, FMV often generates problematic CD results, so they need to be reclassified. A possible approach to reclassify the strongly conflicting pixels is to utilize the spatial connectivity, since the spatial objects are usually spatial-location (neighbourhood) connected.

As shown in Section 3.1, the connectivity in fuzzy topological space includes membership connectivity and location connectivity of points. The former corresponds to the membership domain of points (or pixels), whereas the latter corresponds to the spatial-location domain. The pixels in an image are usually highly correlated with their neighbors. The connectivity of spatial objects therefore mainly depends on the spatial-location structure of points. In order to describe and model the spatial-location (neighbourhood) connectivity of points in fuzzy topological space, the concept of supported connectivity was developed in [30]. The supported connectivity provides a suitable tool to process the fuzzy boundary (i.e., strongly conflicting) pixels.

This study presents a simple but effective method to reclassify the recognized strongly conflicting pixels based on the supported connectivity. The main idea of the method is to search the neighbourhood window of each strongly conflicting pixel and utilize the already labelled neighbouring pixels in the window to reclassify the center pixel of the window. Figure 9a presents an example of a $5 \times 5$ neighbourhood window for a strongly conflicting pixel. $C_{u}\left(C_{c}\right)$ means that the pixel has been already labelled as class $C_{u}\left(C_{c}\right)$. Con indicates that the pixel has strong conflicts remaining to be relabeled. The operational procedure of reclassifying the pixels with strong conflicts includes three steps:

Step 1: Search the location of all the strongly conflicting pixels.

Step 2: For each strongly conflicting pixel $x_{0}$, search its $(2 R+1) \times(2 R+1)$ neighbourhood window and record the already labelled neighbouring pixels in the window, then the pixel $x_{0}$ belongs to the class already assigned to the larger number of neighbouring pixels. When the pixel $x_{0}$ is totally surrounded by the strongly conflicting ones, it will be assigned to class $C_{c}$ if $V_{c}\left(x_{0}\right) \geq V_{u}\left(x_{0}\right)$, or to class $C_{u}$ otherwise.

Step 3: Repeat Step 2 until all the pixels with strong conflicts have been reclassified. 


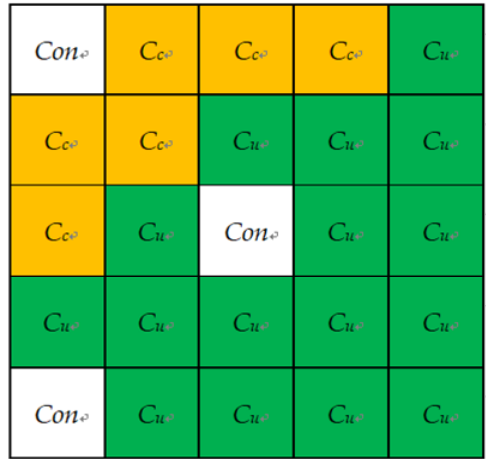

(a)

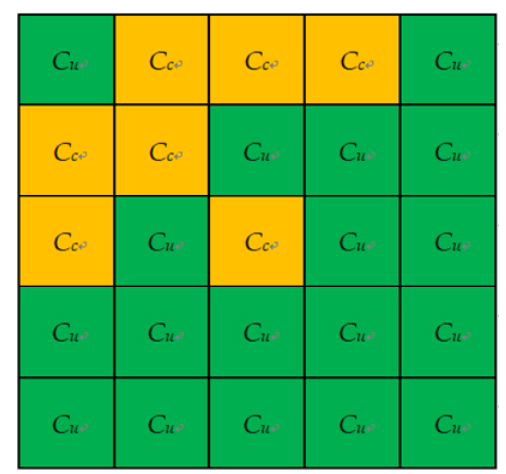

(b)

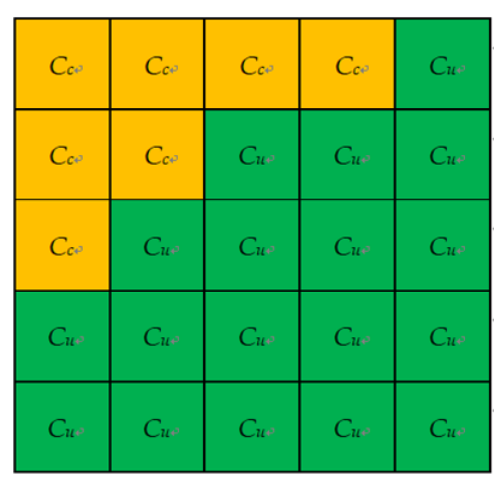

(c)

Figure 9. An example of the changes in topology relations in a $5 \times 5$ neighbourhood window: (a) Partition result by fuzzy topology of (b); (b) CD result of FMV; and (c) CD result after reclassification.

The automatic partition described in Section 3.3 breaks down the topology relations (e.g., the boundary, membership connectivity, and location connectivity) of fuzzy set $F S_{j}(j=u$ or $c)$, which are reconstructed in the reclassification process. Figure 9 shows an example of the changes in topology relations caused by the automatic partition and topology relation reconstruction. The old boundary and location connectivity relations of classes $C_{u}$ and $C_{c}$ (Figure $9 \mathrm{~b}$ ) are broken down by the partition process (Figure 9a), and new boundary and location connectivity relations (Figure 9c) are constructed after reclassification. The Con pixels are relabeled into either $C_{u}$ or $C_{c}$, as shown in Figure 9c.

\section{Results}

\subsection{Experiment Setup and Evaluation Criteria}

In order to evaluate the performance of the proposed FTMV CD method, eleven CD techniques in three types were used as comparative methods (Table 2).

Table 2. Comparison methods used in this study.

\begin{tabular}{ll}
$\begin{array}{l}\text { 1 Four single-DI detectors used to generate the } \\
\text { input of FTMV: }\end{array}$ & CVA, SCM, PCA, and SGD \\
\hline 2 Five similar fusion CD methods: & $\begin{array}{l}\text { The pixel-level fusion approach HFV [17]. } \\
\text { The decision-level fusion approaches MV [19], } \\
\text { DS [19], KMAMV [23], and DSK [24]. }\end{array}$ \\
3 Two advanced non-fusion CD techniques: & $\begin{array}{l}\text { The fuzzy local information C-means } \\
\text { clustering algorithm (FLICM) [33]. } \\
\text { The reformulated FLICM (RFLICM) [6]. }\end{array}$ \\
\hline
\end{tabular}

In the four single-DI detectors, the $\mathrm{CD}$ results were produced by respectively clustering the CVA, SCM, PCA, and SGD DI images with FCM. In the HFV approach, after producing the hybrid DI, the CD was also performed by FCM. FLICM and RFLICM were implemented on the DI produced by the most frequently used CVA technique. The weighting exponent used in FCM, FLICM, and RFLICM was set to 2. The other parameters involved in the approaches were determined according to the trial-and-error procedure. Only the best detection results of each approach were given for the evaluation and illustration of performance.

The CD performance was evaluated based on four accuracy indices: missed detections $(M D)$, the number of changed pixels that are identified as unchanged ones; false alarms $(F A)$, the number of unchanged pixels that are identified as changed ones; overall error $(O E)$, the sum of $M D$ and $F A, O E=M D+F A$; and Kappa coefficient $(K C)$, which are widely used in the CD studies (e.g., $[5,12,19,23,24,34])$. KC is computed by 


$$
K C=\frac{N \times(N C+N U)-((N U+M D) \times(N U+F A)+(N C+F A) \times(N C+M D))}{N^{2}-((N U+M D) \times(N U+F A)+(N C+F A) \times(N C+M D))}
$$

where $N$ represents the number of the pixels in the CD map, and $N U$ and $N C$ denote the correctly detected no-change and change pixels. $M D$ and $F A$ are two specific criteria for evaluating the performance of $C D$ and their importance dependence on the practical application, whereas $O E$ and $K C$ are two global criteria. $K C$ is more cogent than other indices since it involves more classification information $[35,36]$. Additionally, the consumption time $T$ of each algorithm was also recorded for the comparison of time complexity. The eleven comparative algorithms and the proposed FTMV were all carried out in a computer that has Intel(R) Core(TM) i7-9750H 2.59 GHz processor and 16 GB RAM.

\subsection{Experiment Results}

Figures 10 and 11 illustrate the CD maps produced by different algorithms for the Neimeng dataset. Figures 12 and 13 show the CD maps for the Heilongjiang dataset. To illustrate the difference between the CD maps and the corresponding reference image clearly, each CD map was divided into four parts: black and white represent the correctly detected no-change and change pixels, whereas red and yellow denote the $M D$ and FA pixels. For the Hunan dataset, it is too large to clearly exhibit the detailed information of its CD maps in one page. Moreover, the experiment on the Hunan dataset agrees with the results of the other two datasets, so we only present the two best CD maps obtained by DSK and FTMV for the Hunan dataset (Figure 14). Tables 3-5 summarize the five quantitative indices (i.e., $M D, F A, O E, K C$, and $T$ ) of each $C D$ algorithm on the Neimeng, Heilongjiang and Hunan datasets.

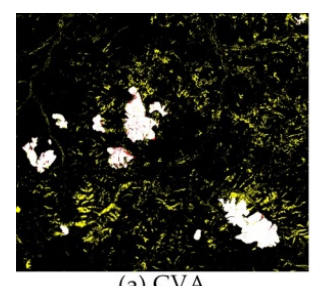

(a) CVA

Legend

$\square$ No Change $\square$ Change

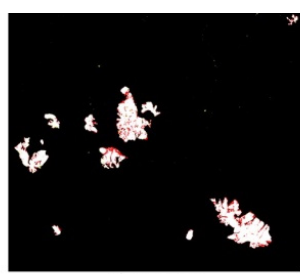

(b) SCM

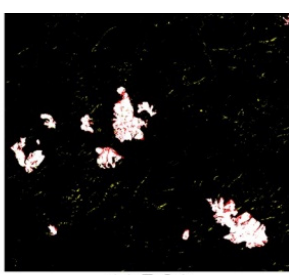

(c) PCA

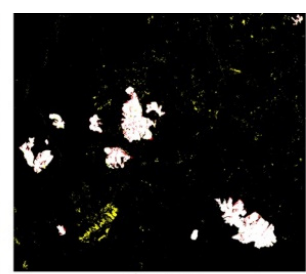

(d) SGD

Figure 10. CD maps created by the four single-DI detectors on the Neimeng dataset.

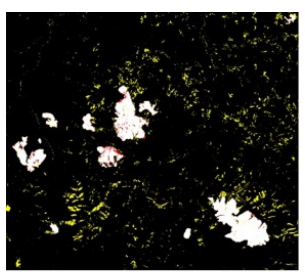

(a) FLICM

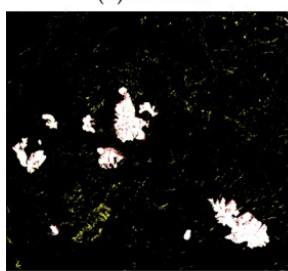

(e) MV

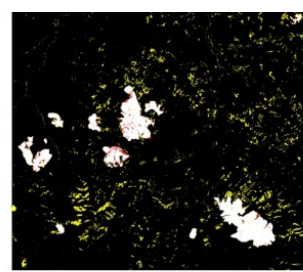

(b) RFLICM

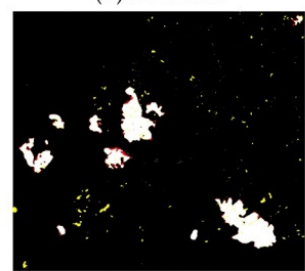

(f) KMAMV

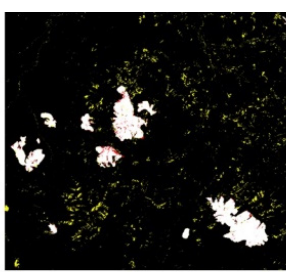

(c) HFV

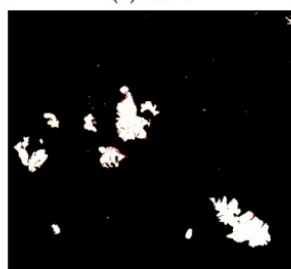

(g) DSK

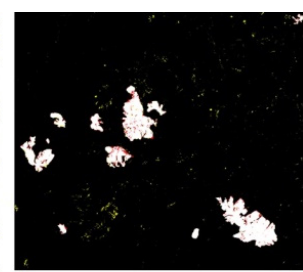

(d) DS

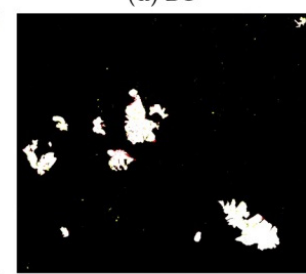

(h) FTMV

Legend

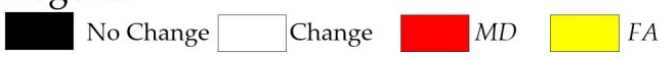

Figure 11. CD maps created by the other eight methods on the Neimeng dataset. 

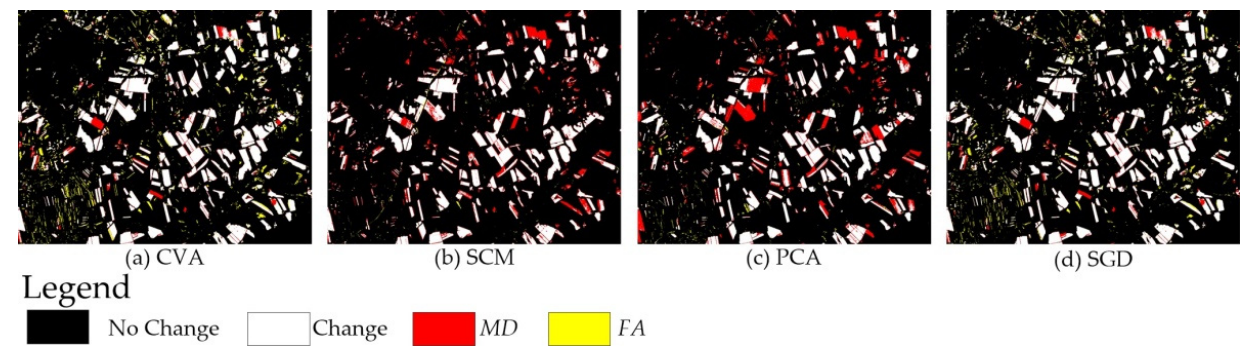

Figure 12. CD maps created by the four single-DI detectors on the Heilongiiang dataset.
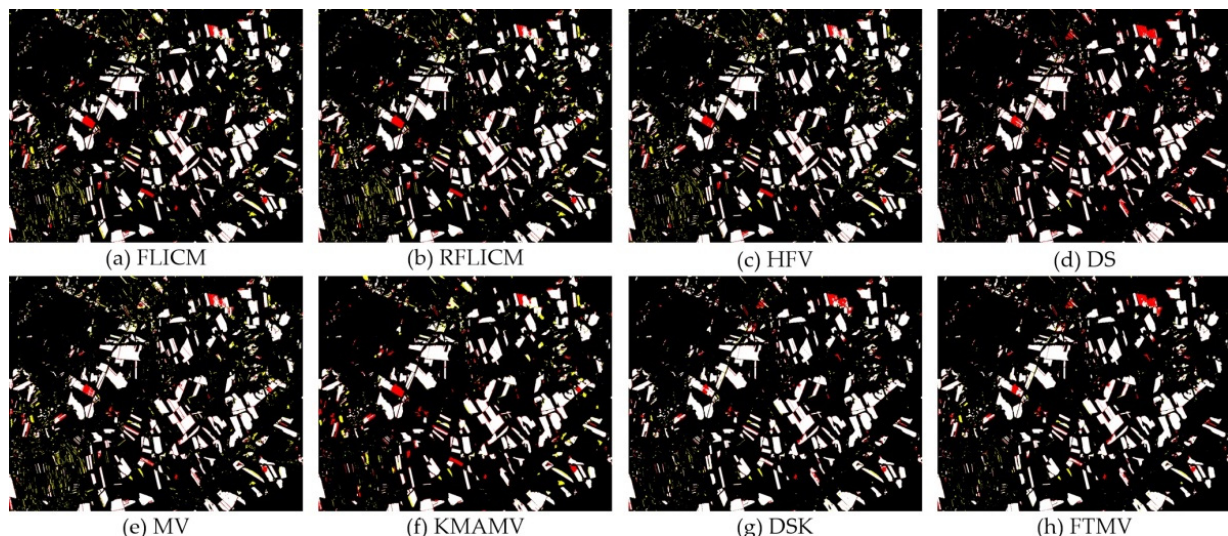

Legend

No Change $\square$ Change

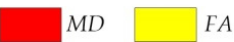

FA

Figure 13. CD maps created by the other eight methods on the Heilongiiang dataset.

Figures 10 and 11 and Table 3 show the qualitative and quantitative comparisons of the CD results provided by different methods of the Neimeng dataset, respectively. Four individual DI detectors created varied but complementary CD maps (Figure 10): The CD maps produced by CVA and SGD have many scattered noise pixels (yellow areas) but few $M D$ errors (red areas), whereas the change map yielded by SCM contains few yellow areas of $F A$ but large red $M D$ areas. This demonstrates the potential for improving the $C D$ performance of single-DI detectors by performing fusion techniques. SCM achieves the best $C D$ results amongst the four individual detectors for the first dataset (Table 3).

For the other seven comparative approaches, FLICM, RLFICM, HFV, and MV generate better CD maps (Figure 11a-c,e) than CVA, but their maps still contain many yellow noise pixels. The CD maps produced by KMAMV and DS are better than those from FLICM, RLFICM, HFV, and MV (Figure 11). However, the maps of KMAMV and DS (Figure 11f,d) are not satisfactory enough in comparison to the reference change image. There still exist apparent scattered yellow $F A$ pixels and a few red $M D$ areas.

The benchmark algorithm DSK (Figure 11g) produces the most accurate CD results among the eleven comparative algorithms, as shown in Figures 10 and 11 and Table 3. Nevertheless, DSK suffers from two serious drawbacks [24] which limit its application: (1) DSK is sensitive to the two key threshold parameters used to recognize the strongly conflicting pixels and requires repeated tuning of them. It is quite laborious and affects the automation level of DSK; and (2) DSK needs to calculate experimental covariance functions for using indicator kriging (to reclassify the pixels with strong conflicts), which is time-consuming and leads to the high computational complexity of DSK.

By introducing MV into fuzzy topological space, the proposed FTMV approach can combine the $\mathrm{CD}$ results from different DIs and largely resolve the conflicting problem during fusion. It achieves significantly better $C D$ results than the first ten benchmark $C D$ algorithms (Figures 10 and 11, and Table 3). For example, the $K C$ value resulted from FTMV is 0.9590 , which is $35.53 \%, 5.23 \%, 8.82 \%, 7.38 \%, 23.18 \%, 24.91 \%, 16.15 \%, 5.41 \%$, 
9.36\%, and 5.79\% larger than CVA, SCM, PCA, SGD, FLICM, RFLICM, HFV, DS, MV, and KMAMV, respectively.

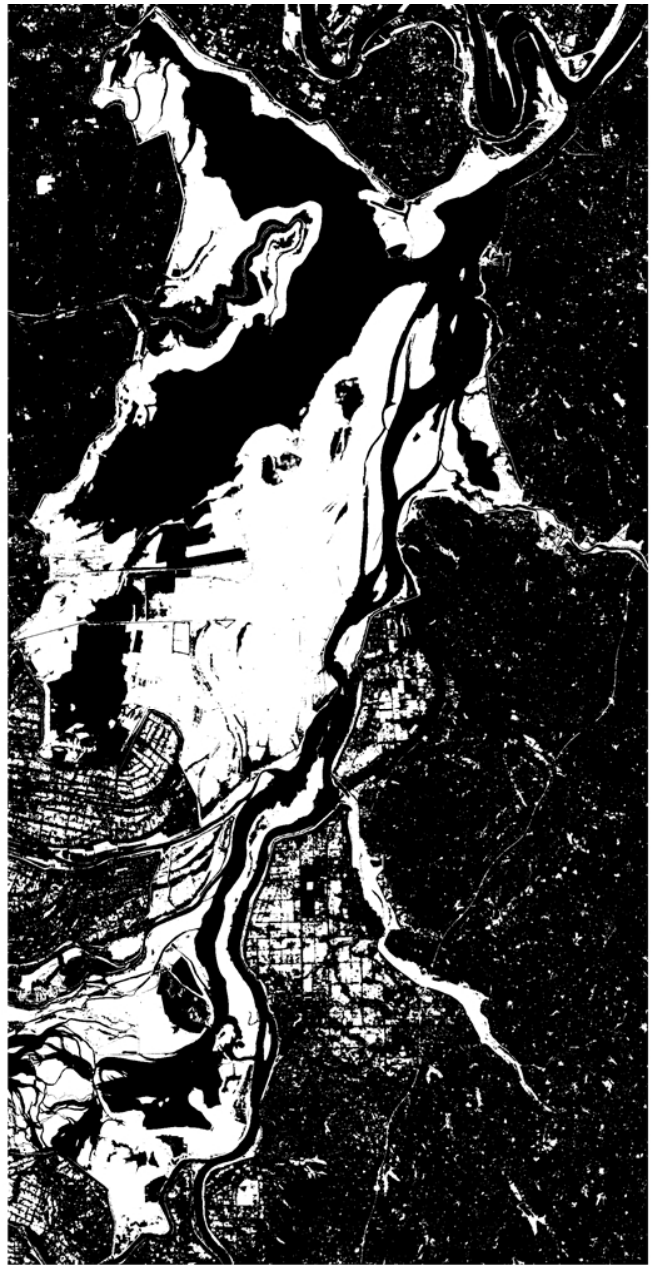

(a) DSK

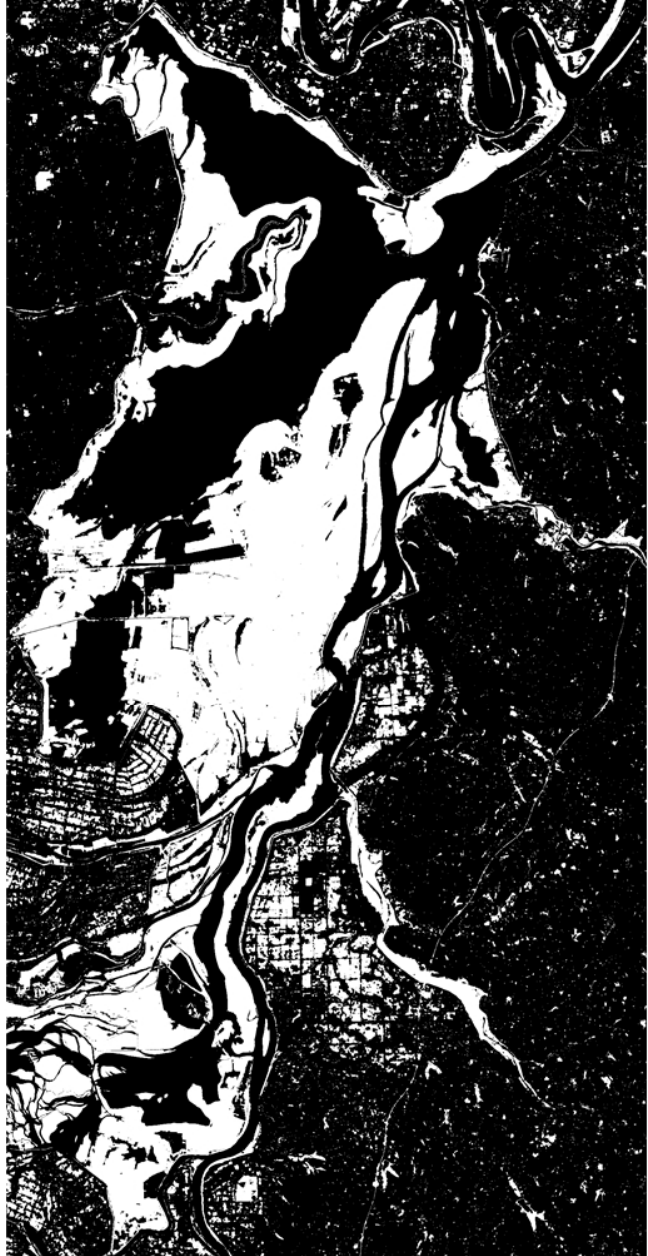

(b) FTMV

Legend

No Change

Change

Figure 14. CD maps created by DSK and FTMV on the Hunan dataset.

Table 3. Quantitative evaluation indices of the Neimeng dataset.

\begin{tabular}{cccccc}
\hline Methods & MD & FA & OE & KC & T/Second \\
\hline CVA & 3400 & 87,835 & 91,235 & 0.6037 & 8.37 \\
SCM & 12,280 & 1174 & 13,454 & 0.9067 & 5.24 \\
PCA & 11,534 & 7989 & 19,523 & 0.8708 & 8.23 \\
SGD & 5521 & 12,956 & 18,477 & 0.8852 & 5.62 \\
\hline FLICM & 4507 & 48,331 & 52,838 & 0.7272 & 29.25 \\
RFLICM & 4356 & 53,102 & 57,458 & 0.7099 & 30.32 \\
HFV & 4594 & 31,556 & 36,150 & 0.7975 & 6.27 \\
DS & 6384 & 8447 & 14,831 & 0.9049 & 29.12 \\
MV & 5703 & 16,354 & 22,057 & 0.8654 & 28.69 \\
KMAMV & 5454 & 10,220 & 15,674 & 0.9011 & $/$ \\
DSK & 3362 & 2528 & 5890 & 0.9616 & 67.42 \\
FTMV & 2790 & 3569 & 6359 & 0.9590 & 33.48 \\
\hline
\end{tabular}


Table 4. Quantitative evaluation indices of the Heilongjiang dataset.

\begin{tabular}{cccccc}
\hline Methods & MD & FA & OE & KC & T/Second \\
\hline CVA & 14,854 & 20,301 & 35,155 & 0.8335 & 2.65 \\
SCM & 34,319 & 1758 & 36,077 & 0.8047 & 2.71 \\
PCA & 41,724 & 3681 & 45,405 & 0.7491 & 2.86 \\
SGD & 15,270 & 14,635 & 29,905 & 0.8555 & 3.13 \\
\hline FLICM & 15,920 & 15,875 & 31,795 & 0.8467 & 10.09 \\
RFLICM & 15,676 & 16,389 & 32,065 & 0.8458 & 11.60 \\
HFV & 14,223 & 14,172 & 28,395 & 0.8631 & 2.95 \\
DS & 23,411 & 3507 & 26,918 & 0.8610 & 14.12 \\
MV & 14,386 & 14,425 & 28,811 & 0.8611 & 13.23 \\
KMAMV & 17,222 & 10,442 & 27,664 & 0.8635 & $/$ \\
DSK & 13,500 & 5264 & 18,764 & 0.9070 & 32.42 \\
FTMV & 14,379 & 5290 & 19,669 & 0.9022 & 15.89 \\
\hline
\end{tabular}

Table 5. Quantitative evaluation indices of the Hunan dataset.

\begin{tabular}{cccccc}
\hline Methods & MD & FA & OE & KC & T/Second \\
\hline CVA & 144,075 & 69,971 & 214,046 & 0.7552 & 15.10 \\
SCM & 217,350 & 9527 & 226,877 & 0.7220 & 13.89 \\
PCA & 196,697 & 24,042 & 220,739 & 0.7345 & 13.30 \\
SGD & 125,686 & 106,307 & 231,993 & 0.7418 & 14.55 \\
\hline FLICM & 159,887 & 49,210 & 209,097 & 0.7564 & 63.85 \\
RFLICM & 148,525 & 59,116 & 207,641 & 0.7607 & 67.76 \\
HFV & 137,523 & 54,602 & 192,125 & 0.7793 & 14.36 \\
DS & 175,590 & 13,446 & 189,036 & 0.7739 & 60.32 \\
MV & 135,334 & 63,804 & 199,138 & 0.7725 & 58.85 \\
KMAMV & 167,081 & 39,183 & 206,264 & 0.7576 & $/$ \\
DSK & 113,873 & 32,437 & 146,310 & 0.8321 & 127.58 \\
FTMV & 120,348 & 27,337 & 147,685 & 0.8295 & 62.39 \\
\hline
\end{tabular}

By comparing with DSK, it is proved that the proposed FTMV method not only overcomes the two shortcomings of DSK, but also provides an result with as good accuracy as DSK. Specifically speaking, FTMV develops an automatic approach to determine the strongly conflicting pixels, whereas DSK requires repeated tuning of two key threshold parameters, thus FTMV has a higher degree of automation and is more feasible in practical applications. Additionally, FTMV reclassifies the recognized strongly conflicting pixels by connectivity analysis, which avoids computing the experimental covariance functions required in DSK. Consequently, the computation time of the proposed FTMV is obviously reduced compared with DSK, as shown in Table 3. It should be pointed out that the recorded time of DSK (in Tables 3-5) only contains its execution time, without the time of manually tuning the two key threshold parameters. The advantages of FTMV over DSK are further discussed in Section 5.3.

As shown in Figures 12 and 13 and Table 4, the CD maps provided by the four singleDI detectors are also complementary for the Heilongjiang dataset (Figure 12): The change maps yielded by CVA and SGD have small red MD areas, whereas the maps from SCM and PCA include few yellow FA errors. SGD performs best amongst the four individual detectors. FLICM and RFLICM generate slightly worse CD results (Figure 13a,b), whereas $\mathrm{HFV}$, DS, MV, and KMAMV produce slightly better CD results (Figure 13c-f), in comparison to SGD (Figure 12d). Again, FTMV and DSK achieve the CD maps (Figure 13g,h) closest to the corresponding reference image, demonstrating their highest accuracy. Compared with DSK, the proposed FTMV CD algorithm achieves nearly identical accuracy but in a more efficient and automated manner (as shown in Table 4 and Section 5.3).

The quantitative indicators obtained by different algorithms on the Hunan dataset (Table 5) were computed according to the fifteen selected image blocks (Figure 4c). As 
reported in Table 5, CVA achieves the lowest $O E$ and highest $K C$ for the four single-DI detectors. FLICM, RFLICM, HFV, DS, MV, and KMAMV show slightly better performance than CVA. For the Hunan dataset, which is quite challenging, FTMV and DSK also produce the best and almost the same accuracy CD results. On the other hand, the proposed FTMV method outperforms DSK in terms of both efficiency and automation (Table 5 and Section 5.3).

\section{Discussion}

\subsection{Robustness of Neighbourhood Window Size}

There is only one parameter (i.e., the parameter $R$ used to control the neighbourhood window size) that needs to be set in the proposed method. This subsection discusses the robustness of parameter $R$. To this end, $R$ was set to 1,2,3,4, and 5, and the cogent $K C$-criterion was applied to assess the $\mathrm{CD}$ performance.

Figure 15 shows the change trends of the $K C$-values versus the parameter $R$ for the three datasets. For all three cases, the $K C$-value increases slowly at first and then decreases slightly when the parameter $R$ increases from 1 to 5 . The $K C$-values are stable for various $R$-values in the set $\{1,2,3,4,5\}$. The stability demonstrates that the proposed FTMV is robust to the parameter $R$ to a large extent. For all the three used datasets, one can choose any value from the set $\{1,2,3,4,5\}$ to achieve a promising $K C$-value of FTMV.

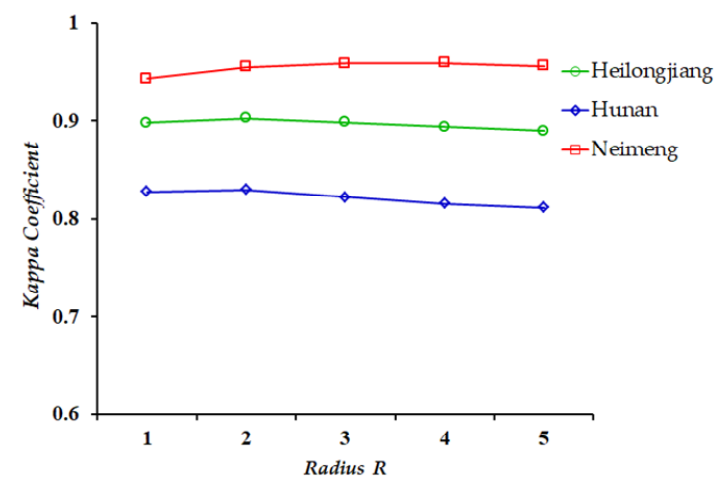

Figure 15. Relationship curves between $K C$-values and parameter $R$.

\subsection{Effectiveness of the Proposed AAM}

This study proposes an automatic and adaptive method (i.e., AAM) to determine $\beta_{u}$ and $\beta_{c}$, which are used in fuzzy topology for automatically recognizing the strongly conflicting pixels. To evaluate the effectiveness of AAM, experimental studies were carried out on the three used datasets. Table 6 presents the $\beta_{u^{-}}$and $\beta_{c}$-values achieved by the proposed AAM and their optimal values. The optimal $\beta_{u^{-}}$and $\beta_{c}$-values were obtained by parameter tuning. We changed $\beta_{u}$ and $\beta_{c}$ from 0.5 to 1 with an incremental step size of 0.01 . The cogent $\mathrm{KC}$ was used as the criterion.

Table 6. Values of $\beta_{u}$ and $\beta_{c}$ achieved by the proposed AAM and their optimal values.

\begin{tabular}{ccccccc}
\hline & \multicolumn{3}{c}{ Proposed AAM } & \multicolumn{3}{c}{ Optimal Values } \\
& $\boldsymbol{\beta}_{\boldsymbol{u}}$ & $\boldsymbol{\beta}_{\boldsymbol{c}}$ & KC & $\boldsymbol{\beta}_{\boldsymbol{u}}$ & $\boldsymbol{\beta}_{\boldsymbol{c}}$ & KC \\
\hline Neimeng & 0.90 & 0.65 & 0.9590 & 0.89 & 0.69 & 0.9608 \\
Heilongjiang & 0.90 & 0.55 & 0.9022 & 0.92 & 0.51 & 0.9059 \\
Hunan & 0.85 & 0.55 & 0.8295 & 0.88 & 0.51 & 0.8403 \\
\hline
\end{tabular}

As shown in Table 6, the proposed AAM provides almost optimal results for all three cases: The $\beta_{u^{-}}$and $\beta_{c}$-values determined by AAM are very close to the optimal ones yielded by adjusting them manually. The KC-values obtained based on AAM are also very close to the KC-values with optimal $\beta_{u^{-}}$and $\beta_{c}$-values. This verifies the effectiveness of the proposed AAM method for automatically setting the values of $\beta_{u}$ and $\beta_{c}$. 


\subsection{Advantages of FTMV over DSK}

This section further discusses the differences between DSK and the proposed FTMV algorithm and analyzes the advantages of FTMV over DSK. The comparisons of FTMV and DSK were carried out from the following three aspects:

(1) Implementation procedure: The proposed FTMV model is developed by introducing MV into fuzzy topological space, whereas DSK is proposed based on DS theory and indicator kriging. Table 7 shows the implementation steps for FTMV and DSK. On the one hand, the proposed FTMV method includes only four main steps, whereas DSK includes seven. On the other hand, steps 1,2, and 4 of FTMV have lower or the same time complexity than steps 1, 3 and 7 of DSK, which is discussed as item 2, Computational complexity, in the next two paragraphs. More importantly, step 3 of FTMV divides the initial CD map automatically, whereas step 5 of DSK requires tuning two key threshold parameters manually to partition the initial map. The latter is quite laborious. The implementation of FTMV is much easier than DSK.

Table 7. Implementation procedures for FTMV and DSK.

\begin{tabular}{cccc}
\hline & DSK & FTMV \\
\hline 1 & FCM clustering for four DIs & 1 & FCM clustering for four DIs \\
2 & Compute mass functions for four DIs & & $\times$ \\
3 & DS fusion & 2 & FMV fusion \\
4 & $\begin{array}{c}\text { Compute conflict degree for each pixel } \\
\text { Divide initial CD map by manually } \\
5\end{array}$ & 3 & $\times$ \\
6 & $\begin{array}{c}\text { Compute an experimental covariance } \\
\text { tuning two key threshold parameters }\end{array}$ & & $\begin{array}{c}\text { Divide initial CD } \\
\text { map automatically }\end{array}$ \\
7 & Indicator kriging interpolation & 4 & $\times$ \\
\hline
\end{tabular}

(2) Computational complexity: For step 1 in FTMV (or DSK), the FCM clustering is performed on the gray level histogram of DI rather than on pixels. The time complexity of this step is $O(4 Q M) \times 4$ [37], where $Q$ represents the number of gray levels and $M$ denotes the mean numbers of the four DIs' iterations. The time complexities of steps 2, 3, 4, 5, 6, and 7 in DSK are $O(12 N), O(4 N), O(6 N), O(3 N), O(32 R N)$, and $O\left(2\left(4 \mathrm{R}^{2}+4 \mathrm{R}+1\right) N \times P\right)$, respectively, where $N$ denotes the number of pixels in the dataset used, $R$ denotes the parameter used to control the neighbourhood window size, and $P$ denotes the proportion of the recognized strongly conflicting pixels in all pixels. The time complexities of steps 2, 3 , and 4 in FTMV are $O(3 N), O(11 N)$, and $O\left(2\left(4 \mathrm{R}^{2}+4 \mathrm{R}+1\right) N \times P\right)$, respectively. Summing the time complexity of all steps, the ratio of the computational complexity of DSK to the one of FTMV can be computed as

$$
\operatorname{Rac}(D S K, F T M V) \approx \frac{16 Q M+\left(25+32 R+2\left(4 R^{2}+4 R+1\right) P\right) N}{16 Q M+\left(14+2\left(4 R^{2}+4 R+1\right) P\right) N} .
$$

The DI images are represented in 8-bit gray scale, and thus $Q$ is equal to 256. Since the maximum number of iterations used in FCM was set to 1000, $M$ must be no larger than 1000. According to the experiments in this study, the value of $P$ is about $10 \%$. Here, we set $R$ as 3, which can usually make both DSK and FTMV obtain a promising $K C$-value, for computing $\operatorname{Rac}(D S K, F T M V)$. The $\operatorname{Rac}(D S K, F T M V)$ value will increase with the value of $N$. Usually, the number of pixels in DI is at least $10^{5}$. When $N=10^{5}$, the Rac is approximately 5:2. Theoretically, when $N$ is sufficiently large, $16 Q M$ can be ignored, and the Rac(DSK, FTMV) will approach 5:1. Therefore, the proposed FTMV has much lower computational complexity than DSK, which also can be seen from Tables 3-5 (in which the recorded time of DSK only contains its execution time, without the time of manually adjusting its two key threshold parameters in step 5).

(3) Parameter setting: Only one parameter is needed in the proposed FTMV approach (namely the parameter $R$ ), whereas three parameters are required in DSK (namely the 
thresholds $T_{u}$ and $T_{c}$ and parameter $R$ [24]). Both FTMV and DSK are robust to the parameter $R$ to some extent, as shown in Section 5.1 and literature [24]. However, for the two parameters $T_{u}$ and $T_{c}$ only used in DSK, DSK is sensitive to them and requires repeated tuning of them [24]. This is not only laborious but also affects the automation level of DSK.

From the above comparisons and the experimental results in Section 4.2, it is clear that, in comparison to DSK, the proposed FTMV CD algorithm can guarantee high accuracy while operating in a more feasible manner: (1) It is much easier to implement; (2) it has far lower time complexity; and (3) it has a higher degree of automation.

\subsection{Future Research}

Our experiments have shown that the proposed FTMV CD method performs better than the eleven benchmark methods for all three cases. Furthermore, FTMV gains robustness and generality to different types of Landsat images and changes.

Future work will focus on the following two directions. (1) Other kinds of remotely sensed data will be investigated to further test the performance of FTMV, such as highresolution remote sensing images. To this end, the strategy for producing DI sets needs to be modified based on the characteristics (e.g., resolution and sensor types) of the considered images. (2) In order to further automate FTMV, additional research will be carried out on the automatic determination of the parameter $R$ used in FTMV.

\section{Conclusions}

In this paper, an FTMV fusion method to unsupervised CD is developed and applied to multispectral remotely sensed images. The proposed FTMV model introduces MV into fuzzy topological space. An initial fusion CD map is first produced by combining the CD results from different DI images using an improved MV (i.e., FMV). Then, through the fuzzy topology induced by a level cut interior operator, the initial CD map is automatically segmented into two parts: a weakly conflicting part and strongly conflicting part. Finally, the pixels with weak conflicts are classified as the current class, and the strongly conflicting pixels are relabeled according to connectivity analysis. FTMV is able to integrate the advantages of different $\mathrm{CD}$ results and largely resolve the conflicting problem during fusion.

Our experimental results on three real remote sensing images have clearly corroborated the effectiveness and superiority of the proposed FTMV approach. For all the cases, FTMV performs better than CVA, SCM, PCA, SGD, FLICM, RFLICM, HFV, DS, MV, and KMAMV in terms of both visual and quantitative measures. In comparison to DSK, FTMV not only produces almost exactly the same $\mathrm{CD}$ accuracy, but also presents the following three advantages: (1) much easier to implement, (2) lower time complexity, and (3) higher level of automation. The proposed FTMV works more efficiently and automated while still providing a promising level of accuracy.

In theory, this paper contributes to the CD development by introducing MV into fuzzy topological space for the first time. In methodology, this work provides a new FTMV fusion algorithm for information fusion, proposes a novel $\mathrm{CD}$ technique, and presents an automatic approach to recognize strongly conflicting pixels.

Author Contributions: Conceptualization, P.S. and W.S.; methodology, P.S. and T.D.; software, P.S. and T.D.; validation, P.S., Z.L. and T.D.; writing-original draft preparation, P.S.; writing-review and editing, W.S., Z.L. and T.D.; supervision, W.S.; project administration, P.S.; funding acquisition, P.S. and W.S. All authors have read and agreed to the published version of the manuscript.

Funding: This research was funded by the National Natural Science Foundation of China, grant number 41901341.

Institutional Review Board Statement: Not applicable.

Informed Consent Statement: Not applicable. 
Data Availability Statement: All datasets presented in this study are available upon request from the corresponding author.

Acknowledgments: The authors would like to thank the editors and anonymous reviewers for their valuable comments and suggestions.

Conflicts of Interest: The authors declare no conflict of interest.

\section{References}

1. Hussain, M.; Chen, D.; Cheng, A.; Wei, H.; Stanley, D. Change detection from remotely sensed images: From pixel-based to object-based approaches. ISPRS J. Photogramm. Remote Sens. 2013, 80, 91-106. [CrossRef]

2. Bruzzone, L.; Prieto, D.F. Automatic analysis of the difference image for unsupervised change detection. IEEE Trans. Geosci. Remote Sens. 2000, 38, 1171-1182. [CrossRef]

3. Patra, S.; Ghosh, S.; Ghosh, A. Histogram thresholding for unsupervised change detection of remote sensing images. Int. J. Remote Sens. 2011, 32, 6071-6089. [CrossRef]

4. Shao, P.; Shi, W.Z.; He, P.F.; Hao, M.; Zhang, X.K. Novel Approach to Unsupervised Change Detection Based on a Robust Semi-Supervised FCM Clustering Algorithm. Remote Sens. 2016, 8, 264. [CrossRef]

5. Ghosh, A.; Mishra, N.S.; Ghosh, S. Fuzzy clustering algorithms for unsupervised change detection in remote sensing images. Inform. Sciences 2011, 181, 699-715. [CrossRef]

6. Gong, M.; Zhou, Z.; Ma, J. Change detection in synthetic aperture radar images based on image fusion and fuzzy clustering. IEEE Trans. Image Process. 2012, 21, 2141-2151. [CrossRef] [PubMed]

7. Bovolo, F.; Bruzzone, L.; Marconcini, M. A novel approach to unsupervised change detection based on a semisupervised SVM and a similarity measure. IEEE Trans. Geosci. Remote Sens. 2008, 46, 2070-2082. [CrossRef]

8. Celik, T.; Ma, K.K. Unsupervised change detection for satellite images using dual-tree complex wavelet transform. IEEE Trans. Geosci. Remote Sens. 2010, 48, 1199-1210. [CrossRef]

9. Shi, W.Z.; Shao, P.; Hao, M.; He, P.F.; Wang, J.M. Fuzzy topology-based method for unsupervised change detection. Remote Sens. Lett. 2016, 7, 81-90. [CrossRef]

10. Mura, M.D.; Prasad, S.; Pacifici, F.; Gamba, P.; Chanussot, J.; Benediktsson, J.A. Challenges and Opportunities of Multimodality and Data Fusion in Remote Sensing. Proc. IEEE 2015, 103, 1585-1601. [CrossRef]

11. Du, P.J.; Liu, S.C.; Xia, J.S.; Zhao, Y.D. Information fusion techniques for change detection from multi-temporal remote sensing images. Inform. Fusion 2013, 14, 19-27. [CrossRef]

12. Bruzzone, L.; Prieto, D.F. An adaptive semiparametric and context-based approach to unsupervised change detection in multitemporal remote-sensing images. IEEE Trans. Image Process. 2002, 11, 452-466. [CrossRef]

13. Liu, L.Y.; Jia, Z.H.; Yang, J.; Kasabov, N.K. SAR Image Change Detection Based on Mathematical Morphology and the K-Means Clustering Algorithm. IEEE Access 2019, 7, 43970-43978. [CrossRef]

14. Zheng, Y.G.; Zhang, X.R.; Hou, B.; Liu, G.C. Using Combined Difference Image and k-Means Clustering for SAR Image Change Detection. IEEE Geosci. Remote Sens. Lett. 2014, 11, 691-695. [CrossRef]

15. Bovolo, F. A Multilevel Parcel-Based Approach to Change Detection in Very High Resolution Multitemporal Images. IEEE Geosci. Remote Sens. Lett. 2009, 6, 33-37. [CrossRef]

16. Inglada, J.; Mercier, G. A New Statistical Similarity Measure for Change Detection in Multitemporal SAR Images and Its Extension to Multiscale Change Analysis. IEEE Trans. Geosci. Remote Sens. 2007, 45, 1432-1445. [CrossRef]

17. Zhuang, H.F.; Deng, K.Z.; Fan, H.D.; Yu, M. Strategies Combining Spectral Angle Mapper and Change Vector Analysis to Unsupervised Change Detection in Multispectral Images. IEEE Geosci. Remote Sens. Lett. 2016, 13, 681-685. [CrossRef]

18. Le Hegarat-Mascle, S.; Seltz, R. Automatic change detection by evidential fusion of change indices. Remote Sens. Environ. 2004, 91, 390-404. [CrossRef]

19. Du, P.J.; Liu, S.C.; Gamba, P.; Tan, K.; Xia, J.S. Fusion of Difference Images for Change Detection Over Urban Areas. IEEE J. Sel. Top. Appl. Obs. Earth Remote Sens. 2012, 5, 1076-1086. [CrossRef]

20. Longbotham, N.; Pacifici, F.; Glenn, T.; Zare, A.; Volpi, M.; Tuia, D.; Christophe, E.; Michel, J.; Inglada, J.; Chanussot, J.; et al. Multi-Modal Change Detection, Application to the Detection of Flooded Areas: Outcome of the 2009-2010 Data Fusion Contest. IEEE J. Sel. Top. Appl. Obs. Earth Remote Sens. 2012, 5, 331-342. [CrossRef]

21. Liu, S.; Du, Q.; Tong, X.; Samat, A.; Bruzzone, L.; Bovolo, F. Multiscale Morphological Compressed Change Vector Analysis for Unsupervised Multiple Change Detection. IEEE J. Sel. Top. Appl. Obs. Earth Remote Sens. 2017, 10, 4124-4137. [CrossRef]

22. Hedjam, R.; Kalacska, M.; Mignotte, M.; Nafchi, H.Z.; Cheriet, M. Iterative Classifiers Combination Model for Change Detection in Remote Sensing Imagery. IEEE Trans. Geosci. Remote Sens. 2016, 54, 6997-7008. [CrossRef]

23. Lv, Z.Y.; Liu, T.F.; Shi, C.; Benediktsson, J.A.; Du, H.J. Novel Land Cover Change Detection Method Based on k-Means Clustering and Adaptive Majority Voting Using Bitemporal Remote Sensing Images. IEEE Access 2019, 7, 34425-34437. [CrossRef]

24. Shao, P.; Shi, W.; Hao, M. Indicator-Kriging-Integrated Evidence Theory for Unsupervised Change Detection in Remotely Sensed Imagery. IEEE J. Sel. Top. Appl. Obs. Earth Remote Sens. 2018, 11, 4649-4663. [CrossRef]

25. Mase, S. GeoStatistics and Kriging Predictors. In International Encyclopedia of Statistical Science; Springer: Berlin/Heidelberg, Germany, 2011; pp. 609-612. 
26. Chen, J.; Lu, M.; Chen, X.; Chen, J.; Chen, L. A spectral gradient difference based approach for land cover change detection. ISPRS J. Photogramm. Remote Sens. 2013, 85, 1-12. [CrossRef]

27. Nemmour, H.; Chibani, Y. Multiple support vector machines for land cover change detection: An application for mapping urban extensions. ISPRS J. Photogramm. Remote Sens. 2006, 61, 125-133. [CrossRef]

28. Falco, N.; Mura, M.D.; Bovolo, F.; Benediktsson, J.A.; Bruzzone, L. Change Detection in VHR Images Based on Morphological Attribute Profiles. IEEE Geosci. Remote Sens. Lett. 2013, 10, 636-640. [CrossRef]

29. Lv, Z.; Liu, T.; Benediktsson, J.A.; Falco, N. Land Cover Change Detection Techniques: Very-High-Resolution Optical Images: A Review. IEEE Geosci. and Remote Sens. Mag. 2021, 2-21. [CrossRef]

30. Liu, K.; Shi, W. Computing the fuzzy topological relations of spatial objects based on induced fuzzy topology. Int. J. Geogr. Inf. Sci. 2006, 20, 857-883. [CrossRef]

31. Kittler, J.; Hatef, M.; Duin, R.P.W.; Matas, J. On combining classifiers. IEEE Trans. Pattern Anal. Mach. Intell. 1998, 20, 226-239. [CrossRef]

32. Bezdek, J.C. Pattern Recognition with Fuzzy Objective Function Algorithms; Plenum: New York, NY, USA, 1981.

33. Ma, J.; Gong, M.; Zhou, Z. Wavelet fusion on ratio images for change detection in SAR images. IEEE Geosci. Remote Sens. Lett. 2012, 9, 1122-1126. [CrossRef]

34. Lv, Z.; Liu, T.; Shi, C.; Benediktsson, J.A. Local Histogram-Based Analysis for Detecting Land Cover Change Using VHR Remote Sensing Images. IEEE Geosci. Remote Sens. Lett. 2021, 18, 1284-1287. [CrossRef]

35. Lv, Z.; Li, G.; Jin, Z.; Benediktsson, J.A.; Foody, G.M. Iterative Training Sample Expansion to Increase and Balance the Accuracy of Land Classification From VHR Imagery. IEEE Trans. Geosci. Remote Sens. 2021, 59, 139-150. [CrossRef]

36. Gong, M.; Su, L.; Jia, M.; Chen, W. Fuzzy clustering with a modified MRF energy function for change detection in synthetic aperture radar images. IEEE Trans. Fuzzy Syst. 2014, 22, 98-109. [CrossRef]

37. Cai, W.L.; Chen, S.C.; Zhang, D.Q. Fast and robust fuzzy c-means clustering algorithms incorporating local information for image segmentation. Pattern Recognit. 2007, 40, 825-838. [CrossRef] 